\title{
Affirmation et décolonisation : la cérémonie de rapatriement par la France des toi moko à la Nouvelle-Zélande en perspective
}

\section{Natacha Gagné}

\author{
CpenEdition \\ Journals \\ Édition électronique \\ URL : http://journals.openedition.org/jso/6674 \\ DOI : $10.4000 /$ jso.6674 \\ ISSN : $1760-7256$ \\ Éditeur \\ Société des océanistes \\ Édition imprimée \\ Date de publication : 30 juin 2012 \\ Pagination : 5-24 \\ ISBN : 978-2-85430-032-1 \\ ISSN : 0300-953x
}

Référence électronique

Natacha Gagné, «Affirmation et décolonisation : la cérémonie de rapatriement par la France des toi moko à la Nouvelle-Zélande en perspective », Journal de la Société des Océanistes [En ligne], 134 | 1er semestre 2012, mis en ligne le 12 juillet 2012, consulté le 30 avril 2019. URL : http:// journals.openedition.org/jso/6674 ; DOI : 10.4000/jso.6674 


\section{Affirmation et décolonisation : la cérémonie de rapatriement par la France des toi moko à la Nouvelle-Zélande en perspective ${ }^{1}$}

par

Natacha GAGNÉ*

\section{RÉSUMÉ}

En 2003, le gouvernement néo-zélandais a adopté une politique visant à faciliter le rapatriement des kōiwi tangata (restes humains) māori qui font partie des collections de musées à l'étranger. À la suite de la mise en auvre de la Loi $\mathrm{n}^{\circ}$ 2010-501 du 18 mai 2010 visant à autoriser la restitution par la France des têtes māori à la Nouvelle-Zélande et relative à la gestion des collections, deux cérémonies de rapatriement de toi moko (têtes momifiées tatouées) ont eu lieu sur le sol français. Une de ces cérémonies se tint le 23 janvier 2012 au musée du quai Branly. C'est à l'analyse de cette cérémonie qu'est consacré cet article qui fait ressortir son intérêt symbolique et politique et la situe dans le mouvement plus large d'affirmation et de décolonisation des Māori.

MoTS-CLÉS : Rapatriement, toi moko, Māori, Nouvelle-Zélande, France, décolonisation

Depuis 2003, une décision du Cabinet du gouvernement néo-zélandais a adopté une politique visant à faciliter le rapatriement des kōiwi tangata $^{2}$ (restes humains, littéralement os humains, généralement traduit de nos jours par restes ancestraux) māori qui font partie des collections de musées à l'étranger. Cette décision répond

\begin{abstract}
In 2003, the government of New Zealand adopted a policy to facilitate the repatriation of Māori kōiwi tangata (human remains) housed in foreign museum collections. After the implementation on May 18 th 2010 , of Law no 2010-501, which authorizes France's restitution of Māori heads to New Zealand (and pertains to the management of collections), two toi moko (tattooed mummified heads) repatriation ceremonies took place on French soil. One of these ceremonies was held on January 23 $3^{\text {rd }}, 2012$ at the musée du quai Branly. The present article is built upon an analysis of this ceremony, emphasizing its symbolic and political significance, and situating it within the larger movement of self-affirmation and decolonization of the Māori people.
\end{abstract}

KeYwords : Rapatriation, toi moko, Māori, New Zealand, France, Decolonization

aux demandes exprimées par les Māori dans un contexte plus large de renaissance culturelle et $\mathrm{du}$ processus de décolonisation (interne) en cours depuis les années 1970, et s'inscrit dans le cadre général de la politique de biculturalisme en vigueur en Nouvelle-Zélande. Elle est une réponse aux efforts de la population autochtone

1. Mon séjour de recherche à Paris en janvier 2012 fut rendu possible grâce à une subvention ordinaire du Conseil de la recherche en sciences humaines du Canada (CRSH). J'aimerais également remercier Guillaume Fontanieu qui m'a gracieusement donné une copie de l'enregistrement sonore qu'il avait fait de la cérémonie du 23 janvier 2012 au musée du quai Branly.

2. Le lecteur doit savoir que le «ng » en langue māori se prononce comme le « gn » en français (par exemple, « agneau ») et le « wh » en māori se prononce comme le «f» en français (par exemple, «feu »).

* Professeur agrégée, Université d'Ottawa (Canada), département de sociologie et d'anthropologie, directrice de recherche au CiRCEM (Centre interdisciplinaire de recherche sur la citoyenneté et les minorités), natacha.gagne@uottawa.ca 
pour retrouver le contrôle de son patrimoine et de sa destinée.

Parmi les kōiwi tangata, on retrouve les fameux toi moko (aussi appelés moko mokai ou upoko tuhi), les têtes momifiées/séchées et tatouées d'origine māori qui ont tant fait parler d'elles en France et ailleurs ces dernières années. Ces têtes furent l'objet d'une attention internationale dans les années 1980, quand certaines d'entre elles furent mises aux enchères à Londres. Simpson (2001 : 235) et Paterson (1999: 125) rappellent qu'en 1983, le Marquis de Tavistock retira des enchères un toi moko qu'il avait en sa possession après que le gouvernement de la Nouvelle-Zélande lui en ait fait la demande. Le toi moko fut subséquemment restitué à la Nouvelle-Zélande ${ }^{3}$. Un autre toi moko est devenu célèbre après qu'une décision de la High Court of New Zealand dans l'affaire Re Estate of Tupuna Maori dont le jugement fut rendu en 1988 ait accepté le principe selon lequel la vente ou l'achat de restes humains en vue de profits et comme objet de curiosité est désormais jugé répugnant (abhorrent) par les Néo-Zélandais (Paterson, 1999 : 126 ; Barker, 2006 : 147). La décision permit au président du New Zealand Maori Council, Graham Latimer, de demander une injonction pour empêcher la vente du toi moko. La tête fut retirée de la vente aux enchères qui devait avoir lieu à Londres, et elle fut retournée à la Nouvelle-Zélande. Son destin fut confié aux Māori de façon à ce qu'elle reçoive un enterrement convenable dans le respect des traditions māori. Cette affaire fit en sorte que les grandes maisons de vente aux enchères internationales n'acceptent plus de restes humains d'origine autochtone pour leurs ventes, incluant des toi moko (Paterson, 1999 : 126).

En 1990, lors de la «Taonga Maori Conference $»^{4}$, les participants māori convinrent que les toi moko disséminés à travers le monde devaient être rapatriés. Ils s'entendirent égale- ment pour que les taonga qui se trouvent dans les collections à l'étranger puissent être conservés par les musées, mais à la condition que les visiteurs māori puissent y avoir accès de façon à encourager une relation active avec les propriétaires spirituels māori de ces taonga (Terrell, Wisse et Philipp, 2008 : 96 ; Simpson, 2001 :236). Cette conférence se tint à la suite des expressions de tristesse manifestés par les kaumātua (aînés) māori pendant la célèbre exposition « Te Maori: Te Hokinga Mai / The Return Home $»^{5}$ qui fut en tournée dans des musées américains et néozélandais entre 1984 et 1987 à propos du fait que des taonga māori reposent dans des musées étrangers (Terrell, Wisse, et Philipp, 2008 : 95).

En 1993, une autre initiative laissa sa marque : les autorités tribales Ngai Tahu firent connaître leur position sur le patrimoine et les restes ancestraux, insistant entre autres choses sur l'idée selon laquelle l'autorité et le contrôle sur les restes ancestraux māori devaient être redonnés à la tribu (Simpson, 2001: 236). Le Scotland Museum and Art Gallery fut le premier à répondre positivement à la politique adoptée par les Ngai Tahu en révisant sa propre politique en 1993, spécifiant que le musée consent « to place its research collection of Maori humain remains under Ngai Tahu management and authority (kaitiakitanga) as specified in the Foundation Principles and Statement of Jurisdiction of their policy » (cité dans Simpson, $2001: 236$ ).

En 2003, il fut donc décidé par le gouvernement néo-zélandais que le Museum of New Zealand Te Papa Tongarewa (Te Papa) ${ }^{6}$ aurait la responsabilité des rapatriements des kōiwi tangata Māori (restes ancestraux māori) ${ }^{7}$ au nom du gouvernement, financement à l'appui (Jones and Whitaker, 2009 : 143). ${ }^{8}$ Les demandes en faveur de la mise en place d'une politique nationale relative au rapatriement des kōiwi tangata vinrent donc des Māori eux-mêmes, et ce, dès les années 1970. Des leaders māori comme Maui

3. NDLR. - Voir aussi ci-dessous la Chronologie concernant les têtes tatouées et momifiées māori...

4. Pour une discussion du terme «taonga » qui peut être traduit de façon générale par « trésors culturels et artistiques, objet précieux māori », voir Mead (1997).

5. Cette exposition fut très importante dans le mouvement de renaissance culturelle māori et la prise de conscience politique (voir McCarthy, 2011, pour des détails sur l'exposition et les changements qu'elle amena). L'exposition fit en sorte de stimuler l'intérêt pour le patrimoine et les mātauranga (connaissances) māori (Te Papa, s.d.). Selon McCarthy (2011 : 55), elle fut un des moments marquants d'une période de décolonisation.

6. En 1992, la National Art Gallery et le National Museum furent fusionnés pour donner naissance au Museum of New Zealand qui devint le Te Papa en 1997. Le musée fut inauguré en 1998 dans un nouvel édifice construit dans le port de Wellington. Comme l'indique McCarthy (2007 : 169), ce musée est né à la fin des années 1980 dans les suites de l'importante exposition «Te Maori » et s'inscrit dans le contexte de la «nouvelle muséologie ». Pour une étude détaillée des pratiques muséales à Te Papa, voir McCarthy (2011).

7. Il est également responsable du rapatriement des koimi Moriori (restes ancestraux moriori). Les Moriori sont les autochtones des îles Chatham, un petit archipel à l'est des deux îles principales de la Nouvelle-Zélande et sous sa juridiction.

8. Voir également la section «Rapatriation » du site internet de Te Papa à l'adresse suivante : http://www.tepapa.govt.nz/ aboutus/repatriation/Pages/overview.aspx (consulté le 31 mars 2012). 
Pomare $^{9}$ - qui consacra une partie de sa vie aux rapatriements internationaux et à la création d'un endroit approprié, un wāhi tapu (lieu, dépôt sacré), pour garder les kōiwi tangata à l'intérieur de ce qui s'appelait encore à l'époque le National Museum (Te Papa, s.d.) - et Dalvanius Prime ${ }^{10}$ furent à l'origine de ces demandes (McCarthy, 2011 : 217). Maui Pomare fut responsable du rapatriement des restes de trente-sept ancêtres (Karanga Aotearoa 2012).

Les rapatriements sont sous l'égide d'une unité interne à Te Papa appelé Karanga Aotearoa ${ }^{11}$, programme chargé d'inviter les institutions qui possèdent des kōiwi tangata hors de NouvelleZélande à les rapatrier. Quand les institutions manifestent une ouverture, les responsables du programme s'engagent dans des pourparlers en vue de soumettre un cas de rapatriement aux institutions gouvernementales concernées (Karanga Aotearoa 2012). Une fois que les kōiwi tangata sont retournés en Nouvelle-Zélande, Te Papa les conservent dans son wāhi tapu de façon temporaire, en attendant qu'ils soient rendus à leurs communautés d'origine après que des recherches les aient dûment identifiés ${ }^{12}$.

À la suite des développements mentionnés précédemment et de plusieurs wānanga (séminaires) tenus depuis 1998 avec des représentants tribaux sur le soin à apporter aux kōiwi tangata et à leur gestion, il est entendu que les $i w i$ (tribus) doivent être consultés et doivent participer activement aux processus de rapatriement ${ }^{13}$. Les restes ancestraux māori rapatriés ne sont pas acquis par Te Papa et ne font pas partie de ses collections (Karanga Aotearoa 2012). Ils y sont entreposés de façon provisoire et une politique du musée prohibe leur exposition au public. Un document rédigé en janvier 2012 indiquait que Te Papa avait rapatrié les restes de près de 190 ancêtres en provenance de 14 pays et on estime à environ 500, le nombre de kōiwi tangata māori « still awaiting their homecoming» (Karanga Aotearoa 2012) ${ }^{14}$.

Les restitutions de kōiwi tangata māori par diverses institutions étrangères sont l'occasion de cérémonies de rapatriement. Ce fut le cas en mai 2011 lors de la restitution par la France d'un premier toi moko, celui du Muséum d'histoire naturelle de Rouen. Le 23 janvier 2012, s'est tenue au musée du quai Branly une seconde cérémonie de restitution sur le sol français pendant laquelle 20 toi moko, le plus grand groupe de restes ancestraux jamais rapatriés en une même occasion, furent restitués à la NouvelleZélande. Ces toi moko provenaient des collections du Muséum national d'histoire naturelle, du Musée national de la Marine, du musée du quai Branly, du Muséum de Nantes, du Museum de Lille, du Musée des Beaux-Arts de Dunkerque, du Musée des Confluences, du Musée des Sens, du Musée d'Arts Africains, Océaniens, Amérindiens de Marseille et de l'Université de Montpellier. La cérémonie se tint à l'occasion de la clôture de l'exposition itinérante $E T \bar{u}$ Ake : Standing Strong (Se tenir debout, avoir une position ferme) en provenance de Te Papa et rebaptisée en français Māori : leurs trésors ont une âme. Ces restitutions s'inscrivent dans le cadre de la mise en œuvre de la loi $\mathrm{n}^{\circ} 2010-501$ du 18 mai 2010 visant à autoriser la restitution par la France des têtes māori à la Nouvelle-Zélande et relative à la gestion des collections. Cette loi a rendu possible la restitution à la Nouvelle-Zélande des têtes māori tatouées en organisant la dérogation à la loi $n^{\circ} 2002-5$ du 4 janvier 2002 relative aux musées de France en vertu de laquelle :

«les biens constituant les collections des musées de France appartenant à des personnes publiques font partie de leur domaine public et sont, à ce titre, inaliénables. » (article 11, alinéa II)

Après ce bref survol des processus de rapatriement concernant les restes ancestraux en cours en Nouvelle-Zélande, je propose, dans le reste de cet article, de m'attarder à la cérémonie de rapatriement qui se tint au musée du quai Branly le 23 janvier 2012 à laquelle j'ai eu la chance de participer. Celle-ci avait une couleur toute māori. Avant d'y regarder de plus près, je rappellerai d'abord la structure « traditionnelle » ou la séquence rituelle des hui, une expression générale māori pour parler de n'importe quelle forme de

9. Descendant de Maui Pomare (1876-1930), médecin et politicien influent, il fut entre autre président du National Museum of New Zealand Council.

10. Chanteur, auteur-compositeur et producteur māori, ardent militant du rapatriement des toi moko logés dans les musées et les collections à l'étranger (voir Pickmere, 2002).

11. Pour la politique interne du musée relativement au kōiwi tangata, voir http://www.tepapa.govt.nz/ SiteCollectionDocuments/AboutTePapa/Repatriation/DraftKoiwiTangataPolicy1October2010.pdf (consulté le 31 mars 2012). Une traduction du nom de ce programme serait « Un appel de bienvenue de la Nouvelle-Zélande ». Le karanga est l'appel cérémoniel fait par une femme (habituellement âgée) pour appeler les visiteurs, mais également les esprits des ancêtres sur le marae lors d'un rituel de rencontre (Salmond, 1975).

12. Voir Te Papa (s.d.) sur les règles entourant le wāhi tapu.

13. Sur les demandes formulées par des autorités tribales avant 1998, voir Simpson (2001:236).

14. Pour une liste complète des rapatriements à l'international qui ont eu lieu depuis 1990, voir http://www.tepapa govt.nz/AboutUs/Repatriation/Pages/InternationalRepatriations.aspx (consulté le 31 mars 2012). 
rencontre, mais qui réfère le plus souvent aux rencontres cérémonielles qui se tiennent sur les marae (lieu de rencontre māori et centre cérémoniel) (Salmond, $1975: 1$ ). Je décrirai ensuite dans ses grandes lignes le déroulement de la cérémonie de rapatriement qui dura une heure. Comme nous le verrons, la comparaison entre la structure rituelle « traditionnelle » et celle de la cérémonie de rapatriement est intéressante à plusieurs égards, tant au plan symbolique que politique. Je proposerai dans la dernière partie de ce texte quelques pistes de réflexion afin de situer cette cérémonie dans le contexte plus large d'affirmation et de décolonisation des Māori.

\section{Structure des rituels de rencontre}

Cet aperçu rapide des rituels de rencontre se base sur l'ouvrage classique de l'anthropologue Anne Salmond, Hui: A Study of Māori Ceremonial Gatherings (1975), ainsi que sur l'ouvrage de Tauroa et Tauroa, Te Marae: A Guide to Customs \& Protocols (1986). J'invite les lecteurs à s'y référer pour plus de détails. D'autres sources ont également été consultées : elles seront indiquées aux endroits appropriés.

Salmond (1975) montre que le marae est le cadre ou la scène dans lequel se tiennent les rencontres cérémonielles. Chez les Māori de Nouvelle-Zélande, le marae consiste en un ensemble clôturé de bâtiments parmi lesquels on retrouve la très caractéristique wharenui, la grosse maison ou maison de rassemblement, une cuisine et une salle à manger. La wharenui - aussi appelée whare tipuna (maison ancestrale), wharehui (maison de rassemblement), whare whakairo (maison sculptée), whare rūnanga (maison du conseil) - représente le cœur du complexe cérémoniel. Elle est habituellement nommée du nom d'un ancêtre. D'ailleurs, non seulement la maison représente l'ancêtre - le faîtage étant sa colonne vertébrale et les chevrons, ses côtes -, elle l'incarne. À l'intérieur de la maison, on est entouré et protégé par l'ancêtre éponyme, par d'autres figures ancestrales associées au groupe et par les dieux (Allen, $2002:$ 48). La maison est d'ailleurs considérée comme un être vivant et dans les discours cérémoniels, on s'y adresse directement comme à un aîné (Allen, 2002 : 48). Si la maison est sculptée et/ou peinte, toutes les formes représentent des figures ancestrales importantes, que ce soit des gens, des dieux ou divers éléments. La maison ancestrale est vue comme un livre d'histoire architectural pour ceux qui y sont liés (Salmond, 1975 : 39; Ihimaera, 1972: 117). Rosenblatt (2011) parle d'une sorte de " grammaire » qui participe à la constitution et à la définition du groupe. C'est d'ailleurs le caractère ancestral et symbolique de la maison qui lui confère son caractère tapu (sacré) ${ }^{15}$. Comme le précise Salmond :

« On the marae, a distinctively Māori vision of New Zealand comes into play, a mythological landscape which includes places like Hawaiki the homeland, and the Underworld or Pō. Time stretches back in genealogical stages to the gods, the migrations to New Zealand, and the ancestors. People are aligned into tribes and sub-tribes, each with its own territory bounded by rivers and mountains. In the rituals, incantations are chanted that were once used for tree-felling, carving or paddling canoes, and the rituals themselves are not much different from those described by the earliest travelers. In the hui [rencontres, rassemblements] on the marae, Maoritanga [traduit par Maoridom en anglais ; Māori culture, univers de sens māori] come into its sharpest focus. » (1975: 2)

Si le marae est d'abord et avant tout un lieu de rassemblement, comme Tauroa et Tauroa l'expliquent :

" [it] is the family home of generations that have gone before. It is the standing place of the present generation and will be the standing place for the generations to come. » (1986 : 19)

ce lieu est aussi qualifié de tūrangawaewae (littéralement, un lieu où poser ses pieds, où se tenir debout), et incarne la connexion à la terre, à Papatūānuku, la Terre-Mère, qui est représentée par la cour cérémonielle. Auparavant, les Māori réservaient d'ailleurs le terme «marae»-ou «marae atea »- à la parcelle de terrain se trouvant juste devant la wharenui (Metge, 1976 : 227). Ses fonctions étaient d'accueillir les visiteurs et de fournir un espace pour la tenue des rituels de rencontre. En anglais, l'expression «marae proper » sert de nos jours à distinguer la cours cérémonielle de l'ensemble du complexe.

C'est donc à travers leur tūrangawaewae, que les Māori tirent leurs droits comme tangata whenua (gens de la terre / du lieu) et maintiennent la connexion aux ancêtres qui fréquentèrent le marae et qui y sont maintenant enterrés. Selon Durie (1999 : 362), le marae est étroitement lié à l'identité, laquelle embrasse des relations beaucoup plus larges, dans le temps et l'espace :

«connections over time, connections between tribes and peoples, connections which link secular and spiritual, temporal and ethereal. » (Durie, 1999 : 359)

15. Les principes de tapu et noa (ordinaire, libre de restrictions religieuses) servent à définir l'ensemble des relations sur le marae et à orienter les rituels. Pour des détails, voir Metge (1976) et Salmond (1975). 
La parcelle de terre associée au marae possède maintenant une importance symbolique très grande puisque pour certains groupes, elle est la seule propriété collective qui leur reste étant donné que les terres māori ont été vendues ou confisquées dans leur majorité durant la période coloniale ${ }^{16}$.

Traditionnellement, les marae étaient soit familiaux, soit tribaux et étaient construits en l'honneur d'un ancêtre de la famille étendue ou de la tribu sur les lieux de sa sépulture. À partir des années 1960, étant donné l'importante migration urbaine ${ }^{17}$, des marae tribaux et pantribaux furent construits en ville pour répondre aux besoins des citadins. Dans certains cas, il s'agissait de marae construits par une tribu et destiné en propre à ses membres pour répondre à leurs besoins loin du marae «traditionnel». Dans d'autres cas, l'initiative de la construction d'un marae fut celle d'un groupe de personnes venant de différentes tribus et ayant besoin d'un lieu pour leurs rassemblements communautaires, leurs funérailles et pour mener à bien certains projets. Ce type de marae acquit une vocation pan-tribale, c'est-à-dire qu'il avait pour objectif de servir l'ensemble ou une partie de la communauté des Māori vivant en ville aux origines tribales diversifiées (voir, entre autres, Rosenblatt, 2002, 2011 ; Tapsell, 2002; Walker, 2004 pour des exemples et plus de détails). Plusieurs institutions publiques telles les écoles, universités, hôpitaux, ministères et agences gouvernementales se dotèrent aussi de marae, souvent à la suite de demandes formulées par des groupes s'inscrivant dans le mouvement d'affirmation māori depuis les années 1960, mouvement largement qualifié de « renaissance culturelle». Dans ce contexte, les marae constituent un symbole important d'identité et de continuité et sont devenus des sites clés pour les Māori de leur identité, de leur mana (pouvoir spirituel, autorité, prestige, statut) et de leur affirmation culturelle et politique et un lien de concertation important en vue d'actions collectives (entre autres, voir Metge, 1976; Rosenblatt, 2005, 2011; Salmond, 1975; Sissons, 2010; Walker, 2004).

La partie cérémonielle d'un hui s'appelle le mihi (accueil). Elle est constituée de plusieurs éléments ou unités rituelles. Je présenterai maintenant très brièvement les principales ${ }^{18}$. Il faut s'imaginer que la scène prend place sur un marae. Un groupe de visiteurs vient d'arriver. Quand ils reçoivent le signal que les hôtes sont prêts à les accueillir, le groupe se resserre et se déplace à la porte d'entrée de l'enceinte du marae. Le rituel est alors sur le point de commencer. Le premier élément du rituel est la waerea (incantation protectrice). Elle consiste en un chant long et continu dont l'exécution par le kaumatua (aîné) en chef débute juste avant l'entrée sur le marae et se poursuit au moment où le groupe commence à avancer. L'idée est d'ouvrir la voie et à la dégager de tout obstacle ou influence surnaturel qui pourrait être néfaste pour le groupe (Salmond, 1975 : 131). Cet élément du rituel est plus rarement présent de nos jours et peut être remplacé par des prières chrétiennes, par exemple (Salmond, 1975 : 132).

Le deuxième élément est le wero (challenge ou défi rituel). Cette partie du rituel est exécutée par un jeune guerrier au torse nu, vêtu d'un piupiu (pagne de lin) qui court, bondit, grogne et grimace en brandissant son arme lorsque les visiteurs mettent le pied sur le marae. La forme moderne du wero est une forme abréviée et ritualisée de la démonstration de force militaire que les hôtes offraient à leurs visiteurs à l'époque où les guerres intertribales étaient fréquentes et où il était impossible de savoir à coup sûr les intentions des visiteurs. Aujourd'hui, cette partie du rituel est accomplie quand un visiteur important s'apprête à entrer sur le marae. C'est :

« a symbolic way of acknowledging his mana [pouvoir spirituel, autorite, prestige, statut], at the same time expressing the strength of the local people. » (Salmond, $1975:$ 135)

L'élément suivant est le karanga (appel de bienvenue). Cet appel est habituellement chanté en premier par une femme âgée du groupe hôte vêtue de noir et agitant des feuillages verts. L'appel a pour but de guider les visiteurs sur le marae. Celle-ci se tient debout sur le porche de la maison de rassemblement ou juste devant celleci, sur la cour cérémonielle. Elle est parfois accompagnée par d'autres femmes. À l'avant des visiteurs marchent également une ou plusieurs femmes âgées, également vêtues de noir, qui vont répondre à l'appel tout en avançant tranquillement. Le karanga est un long appel constitué de

16. Pour des détails sur les ventes et les confiscations des terres, voir Belich (1996).

17. Avec les demandes du marché du travail et les pressions exercées par une importante croissance démographique sur un territoire devenu plutôt restreint à la suite des confiscations foncières, le mouvement de migration des Māori vers la ville débuta dans les années 1930, pour s'accélérer dans les années 1950 et atteindre un sommet dans les années 1960 (Belich, 2001). Leur présence est remarquable aujourd'hui dans le tissu urbain du fait que presque $84,4 \%$ d'entre eux habitent en ville et qu'ils représentent 14,6\% de la population néo-zélandaise (Recensement 2006).

18. Salmond (1975) montre bien que celles-ci se subdivisent à leur tour en divers éléments. 
salutations et d'invocations des morts. Comme l'explique encore Salmond :

" [b]y the time the callers have finished the dead are almost tangibly present on the marae. All the group, living and dead members alike, are brought together, making long unbroken chain of kinsmen that stretches right back to Hawaiki and the Pō (Underworld). » (1975: 140)

Le pōwhiri (chant(s) mimé(s) d'accueil, traduit par action chant (s) en anglais) est le prochain élément du rituel. Comme l'indiquent Tauroa et Tauroa (1986 : 54), le pōwhiri peut parfois ne pas être exécuté du tout ou être exécuté avant le karanga. À un moment où l'autre, les deux éléments vont se chevaucher. Il est exécuté par l'ensemble des hôtes - lesquels sont installés en rangées devant la maison de rassemblement, les femmes les plus âgées devant, suivies des plus jeunes et des hommes derrière - pendant que les visiteurs se rapprochent. Pendant l'exécution du chant, les hôtes vont très souvent tenir des feuillages verts dans leurs mains, en particulier en présence d'un tūpāpaku (dépouille d'une personne décédée) lors d'un tangihanga (funérailles). Leurs mains s'agitent dans une sorte de tremblement en même temps que les bras exécutent des mouvements de bas en haut. Les feuillages représentant te ao et te pō (la lumière et les ténèbres, la vie et la mort) (Tauroa et Tauroa, 1986 : 54). Ils servent aussi de protection contre la contagion de la mort ou des influences maléfiques (Schwimmer, 1965: 155). Tel que précisé par Tauroa et Tauroa (1986: 55), le pōwhiri sert plus généralement à se parer des mauvais esprits qui peuvent être présents tout en s'assurant que les visiteurs traverseront le marae sans danger. Les chants de cette unité rituelle étaient autrefois utilisés par les pagayeurs des waka (pirogues, canots) pour garder un rythme constant (Salmond, 1975 : 143). À travers ces chants, le groupe hôte «tire» métaphoriquement les manuhiri qui représentent le waka à gué en toute sécurité (Matenga-Kohu et Roberts 2006).
«[T]he voices of the powhiri symbolically represent the tow rope by which visitors are pulled safely onto the marae. » (Tauroa et Tauroa, $1986: 56$ )

Cette corde métaphorique s'entremêle à celle tressée par le karanga pour la renforcer et lier ensemble tous les participants au rituel en les mettant sous la protection du tapu (caractère sacré) du marae.

Le tangi (gémissements et pleurs) est un autre élément important du rituel de rencontre. Chaque rassemblement māori est l'occasion de se remémorer les morts et d'exprimer son regret par rapport aux êtres aimés disparus. Alors que le karanga les rappelle à la mémoire :

« [t]he old women dress in black, the colour of mourning, and as they advance into the marae they begin a high, uncanny wail, sometimes beting their breats with a slow, hopeless motion, or waving a handkerchief as they cry. » (Salmond, $1975:$ 145)

Les visiteurs et les hôtes se joignent au tangi. Têtes baissées, ils peuvent laisser couler quelques larmes et quelques gouttes de mucus. Seulement les femmes, cependant, gémissent ; les hommes pleurent en silence (Salmond, 1975 : 146). Lors de tangihanga (funérailles), les gémissements et les pleurs sont particulièrement longs et forts.

Un élément très important du rituel est le whaikōrero (échanges oratoires). Ce moment correspond à l'échange de discours formels. Les orateurs sont des hommes ayant atteint le statut de kaumatua (aîné). Ce statut s'atteint vers la cinquantaine. Des critères comme le niveau de connaissance des traditions, de la langue māori ainsi que le mana (statut, autorité, pouvoir spirituel) entrent alors en ligne de compte. Dans la plupart des tribus, les femmes ne peuvent pas prendre la parole comme oratrice dans cette partie formelle de la rencontre cérémonielle ${ }^{19}$. Certaines tribus font pourtant exception à cette règle pour les femmes de haut statut (pour plus de détails, voir Salmond, $1975: 127$; Tauroa et Tauroa, 1986: 76-77). Un bon orateur est un personnage très estimé : il possède une maîtrise

19. Plusieurs raisons sont données pour expliquer ce choix et celles-ci donnent parfois lieu à certaines controverses et débats (voir, par exemple, Tauroa et Tauroa, 1986 : 76-77, Berry 2005). Hommes et femmes sont vus comme ayant chacun leurs rôles respectifs à jouer, aucune cérémonie ne pouvant se dérouler en l'absence des uns ou des autres dans cette société ambilinéaire. On attribue d'ailleurs aux femmes et aux hommes des mana (statut, autorité, pouvoir spirituel) particuliers, respectivement le mana wahine et le mana tane, qui supposent des rôles et des responsabilités spécifiques, ainsi que des relations particulières au tapu, au domaine du sacré. Ces deux formes de mana sont différentes, mais le mana « générique » étant un principe unitaire et donc indivisible, l'un ne peut aller sans l'autre. Ainsi, pour ne prendre qu'un exemple, si les hommes sont ceux qui prennent la parole dans les protocoles d'accueil, ce sont toujours les femmes qui ont le dernier mot puisque, lorsque les hommes terminent leur allocution pendant la période du whaikōrero, elles se lèvent et chantent (Salmond, 1975: 127). Cette waiata (chanson traditionnelle) qui est choisie avec soin est l'occasion d'appuyer ce que les hommes viennent de dire, de manifester certains désaccords ou de réajuster le tir. Les femmes, par leurs chants, ont d'ailleurs le pouvoir d'arrêter un homme de parler s'il parle trop ou de façon inappropriée. Un autre point important souvent rappelé dans les débats relatifs à l'absence de femmes comme oratrice et au fait qu'elles siègent toujours derrière les hommes lors des rencontres cérémoniels est le suivant : les femmes, étant celles qui portent la vie et assurent la reproduction, ont un plus grand mana. Ce serait donc à cause de cette capacité 


\begin{tabular}{|c|c|c|}
\hline \multirow{7}{*}{ 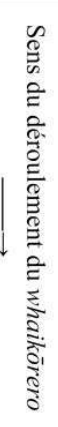 } & Hunga kāinga (hôtes) & Manuhiri (visiteurs) \\
\hline & $\begin{array}{c}\text { Whakaaraara } \\
\text { (appel d'avertissement) }\end{array}$ & $\begin{array}{c}\text { Whakaaraara } \\
\text { (appel d'avertissement) }\end{array}$ \\
\hline & Tau (chant ésotérique) & Tau (chant ésotérique) \\
\hline & Salutations aux visiteurs & $\begin{array}{c}\text { Salutations au marae et à la maison } \\
\text { de rassemblement }\end{array}$ \\
\hline & Salutations aux morts & Salutations aux morts \\
\hline & Salutations aux vivants & Salutations aux vivants \\
\hline & Sujet spécifique & Sujet spécifique \\
\hline
\end{tabular}

FIGURE 1. - Structure de whaikōrero types

de la généalogie, des chants anciens, de l'histoire locale et des proverbes (Salmond, 1975 : 147). Il possède également l'art de la rhétorique et est capable d'effets dramatiques avec ses gestes et mouvements.

Le discours des orateurs suit une structuretype qu'on retrouve dans la figure 1 élaborée à partir de Salmond (1975 : 171). Les salutations diffèrent légèrement selon le groupe auquel appartient l'orateur.

Pendant le whaikōrero, plusieurs orateurs vont prendre la parole à tour de rôle. Tout dépendant du kawa (protocole, règles cérémonielles) en vigueur sur le marae hôte, l'ordre des orateurs va changer. Dans certains cas, les orateurs de chaque groupe parleront en alternance à commencer par les hôtes, tantôt tous les orateurs du côté des hôtes parleront et seront suivis par tous les orateurs du côté des visiteurs. De chaque côté, l'ordre des tours de parole des différents orateurs variera en fonction des traditions propres à chaque région : parfois l'orateur le plus important et le plus doué parle en premier ce qui lui permet de retracer les liens généalogiques entre les deux groupes en présence; parfois il parlera en dernier et sera responsable de l'impression finale que son groupe laissera à l'autre groupe. La prestation de chaque orateur, incluant celle du dernier, est suivie par une waiata (chanson traditionnelle) chantée en chœur par l'orateur et son groupe qui sera choisie en fonction de la circonstance. Le groupe se lève alors en appui à l'orateur et chante sous le leadership des femmes du groupe ${ }^{20}$. Dans le cas du tout dernier orateur - qui est donc du côté des visiteurs - il reprend succinctement la parole après la waiata et dépose un koha (présent, cadeau) sur le marae atea (cour cérémo- nielle) tout près du groupe hôte à son intention, ce qui clôturera cette unité rituelle. Lors du dépôt du koha, le dernier orateur doit donc traverser l'espace qui n'a jamais été traversé par aucun des membres des deux groupes jusqu'à ce moment.

Le moment est alors venu d'entrer dans la dernière phase du rituel : le hongi et le harirū. Le hongi est la forme traditionnelle de salutation māori qui consiste, pour deux personnes qui se rencontrent, à se presser le nez et parfois le front l'un contre l'autre tout en se serrant la main (harirū).

«To hongi, people press noses and foreheads, symbolically representing a meeting and sharing of personal mana [statut, autorité, pouvoir spirituel], mauri [essence, soufflé de vie] and tapu [sacralité]. In doing so, manuhiri [invités] and tangata whenua [hôtes] are reconciled. » (Ngata, 2005 : 34-35)

À l'appel d'un kaumatua (aîné) du côté des hôtes, les siens se lèvent et forment une ligne. Les visiteurs traversent alors la cour cérémonielle pour serrer la main de chacun et/ou faire le hong $i$ et/ou la bise. Cela indique la fin de la partie proprement rituelle de rencontre. L'atmosphère devient alors amicale et détendue puisque le rituel a fait en sorte de lever tous les tapu (interdits, aspects sacrés).

Les invités seront par la suite invités à partager l'hospitalité des hôtes en partageant un repas. Ensuite, les participants à la rencontre pourront s'adonner aux autres activités prévues, lesquelles sont liées au take (objet, cause) spécifique de la rencontre. Salmond (1975: 179) identifie deux grandes classes de take : ceux dont l'événement principal est lié aux étapes importantes de

reproductrice que les femmes devraient être protégées des forces surnaturelles présentes dans les cérémonies sacrées. Ce serait également pour cette raison que ce sont les femmes âgées qui ne sont normalement plus en âge de procréer qui font le karanga et qui marchent en avant du groupe lors de l'entrée sur un marae étranger et que ce sont les hommes de grand mana et donc plus âgés qui s'exposent publiquement, au nom de tous, durant le whaikōrero. La hiérarchie « avant» / " arrière » n'aurait d'ailleurs pas existé dans la langue māori : elle aurait été introduite par la colonisation (Sharples, in Berry, 2005). Seule existerait une distinction en termes spatiaux entre des endroits plus ou moins tapu, c'est-à-dire exposés, dangereux ou risqués.

20. Se rapporter à la note 19 pour les détails de la composition du groupe. 
la vie (funérailles, $21^{\mathrm{e}}$ anniversaire de naissance, mariage, etc.) et qui se focalisent sur un ou des individus et ceux dont l'événement principal implique un groupe. Ces événements de nature plus collective peuvent être, par exemple, l'ouverture d'un nouveau marae, l'accueil d'invités distingués, les rencontres d'associations, des événements en lien avec une Église.

En plus de divisions en fonction du sexe et de l'âge des acteurs, il ressort donc clairement que le rituel de rencontre est ordonné en fonction d'une division entre les hunga kāinga (gens du lieu, hôtes), aussi appelés tangata whenua (gens de la terre / du lieu, autochtone) et les manuhiri (invités ou visiteurs). Les deux groupes restent clairement séparés, occupent des emplacements différents et se font face pendant toute la durée du rituel. Comme le précise Salmond (1975 : 127), ceci est particulièrement clair pendant le whaikōrero (échanges oratoires) puisque les orateurs du groupe local et du groupe en visite prennent la parole soit en alternance, soit en deux blocs qui se suivent. Durant le karanga (appel de bienvenue), l'appel sera lancé par une femme du groupe local à qui répondra une femme du groupe en visite. C'est seulement à la fin du whaikōrero que les deux groupes se rencontrent pour le hongi (action de se presser le nez). En dehors de ces moments, chaque groupe est responsable de parties précises de la séquence rituelle.

\section{La cérémonie de rapatriement au musée du quai Branly du 23 janvier 2012}

Comme dans un hui (rencontre cérémonielle) typique, la cérémonie débuta alors que le silence s'était fait dansl'assistance. Les manuhiri (invités/ visiteurs), dans ce cas la délégation de la Nouvelle-Zélande qui comptait une dizaine de Māori, firent leur entrée au son du pūtātara (conque, trompette en coquillage) dans le théâtre Claude Lévi-Strauss. Un kaitātaki (challenger, jeune guerrier qui fera le wero ou défi rituel) suivi des femmes plus âgées marchaient devant, suivis par les femmes māori plus jeunes puis les hommes māori et enfin, par d'autres membres de la délégation, pas tous d'origine māori. Tous étaient vêtus de noir ou drapés de noir et les femmes māori en tête de la procession portaient des feuillages verts autour de la tête. Parmi le groupe des visiteurs, on comptait Derek Lardelli, kaumatua (aîné), leader de la délégation et membre du groupe d'experts sur le rapatriement au Te Papa, Michelle Hippolite, kaihautū (leader māori) dans l'équipe de direction du Te Papa, et Rosemary Banks, Ambassadeur de Nouvelle-Zélande en France ${ }^{21}$.

Le théâtre prit, pour la durée de la cérémonie, les allures d'un marae ou, exprimé peut-être plus correctement, tint lieu de marae. En effet, comme l'expliquent Tauroa and Tauroa :

«many people use the term 'marae' for any place where they choose to welcome visitors, especially when they choose to use a format corresponding to that of a marae welcome. A room, therefore, may loosely, though acceptably, be referred to as a marae for the purposes of welcoming visitors. » (1986:141)

Selon ce que j'ai pu observer, la même chose peut être dite à propos d'une salle de réception, d'un bureau, d'une pièce d'un hôpital, de la cour arrière d'une maison, ou de la cour d'une école, par exemple, si un karanga (appel cérémoniel de bienvenue) et un échange de mihimihi (salutations cérémonielles, échanges oratoires) ont lieu (Tauroa and Tauroa, 1986 : 141 ; Ngata, 2005 : 32). Les gens présents parleront alors du lieu «comme » d'un marae pendant la durée du rituel de rencontre. On pouvait voir dans la disposition des personnes et des toi moko pendant la cérémonie ainsi que dans la décoration du théâtre Claude Lévi-Strauss des éléments qui évoquaient le marae traditionnel en son absence. J'y reviendrai.

Quant au hunga käinga (gens du lieu, hôtes), ici les Français, ils étaient représentés par des hauts fonctionnaires et des membres du gouvernement dont Frédéric Mitterrand, ministre de la Culture, Catherine Morin-Desailly, sénatrice de la Seine-Maritime et auteure de la proposition de loi concernant la restitution des têtes māori à la Nouvelle-Zélande, et Richard Tuheiava ${ }^{22}$, sénateur de la Polynésie française. Alors que la délé-

21. La délégation néo-zélandaise incluait six artistes māori (dont Derek Lardelli, tatoueur, sculpteur, compositeur et chorégraphe) venus à Paris avec le soutien de l'organisme à but non lucratif qui fait la promotion de l'art māori contemporain, Toi Mãori Aotearoa: Māori Arts New Zealand, pour animer des ateliers durant la fin de semaine de clôture de l'exposition Māori: leurs trésors ont une âme au musée du quai Branly (voir le site de Toi Māori Aotearoa, http://www.maoriart. org.nz/latest_news.php?news_id=59, consulté le 31 mars 2012).

22. Pour en savoir davantage sur le sénateur Tuheiava, son identification comme autochtone et Mā'ohi et ses liens avec les Māori, voir Massau (2011). On peut peut-être se surprendre du fait que les dignitaires français n'aient pas donné une place plus importante au sénateur lors de la cérémonie. Celui-ci aurait probablement pu faire au moins un court kōrero (discours ; 'ōrero en tahitien) en réponse à celui de Derek Lardelli ou aurait à tout le moins pu leur souhaiter la bienvenue en tahitien, une langue qu'il a apprise (voir Massau, 2011 : 28). Dans l'esprit māori, cependant, son jeune âge (37 ans) ne lui conférait pas un statut de kaumatua, et seuls les kaumātua prennent habituellement la parole dans ce genre de cérémonie, une situation qui tend pourtant 
gation de la Nouvelle-Zélande s'avançait vers la scène à partir de l'arrière du théâtre, le groupe hôte attendait les invités en se tenant debout à droite de la scène, disposés sur trois rangées. Sur la première rangée se trouvaient les principaux dignitaires, notamment tous ceux qui allaient prendre la parole. Les hôtes français respectaient donc l'agencement général des rituels de rencontre.

De l'arrière de la salle, les manuhiri s'avancèrent vers leurs hôtes. En chemin, le kaumatua et leader du groupe, Derek Lardelli, prononça d'abord une karakia, une prière en remplacement d'une waerea, une incantation ancienne prononcée auparavant contenant quantité d'archaïsmes et de références obscures, aux dires de Salmond (1975 : 131) en vue d'assurer la protection contre les mauvais esprits et les possibles mākutu (sorcellerie, magie noire).

Puis le groupe se mit à exécuter un pōwhiri (un chant mimé d'accueil), un chant qui est habituellement exécuté par le groupe hôte. Le cri de ralliement du leader «Turuki! Turuki! [croître] » étaient suivi d'une réponse en chœur du reste du groupe «Paneke! Paneke! [avancer] » qui avançait à l'unisson. Ce premier chant de l'unité rituelle, fut suivi par un second chant, qu'on retrouve dans Salmond (1975 : 142), très connu et souvent repris :

\begin{tabular}{|c|c|}
\hline Kümea mai & te waka! ${ }^{23}$ \\
\hline Tirons & le canot \\
\hline Tōia mai! & te waka! \\
\hline Traînons & le canot! \\
\hline Ki te urunga! & te waka! \\
\hline Vers l'oreiller! & le canot! \\
\hline Ki te moenga! & te waka! \\
\hline Vers l'endroit de repos! & le canot! \\
\hline Ki te takotorangi i takoto & ai, te waka e! \\
\hline
\end{tabular}

Ce chant montre bien le parallèle établi entre le groupe et l'arrivée en toute sécurité d'un canot à gué, tel que mentionné dans la partie précédente.

La femme māori la plus âgée du groupe qui avançait en tête de la délégation, exécuta un karanga (appel de bienvenue) immédiatement après les deux chants du pōwhiri, un appel dans lequel elle saluait leurs hôtes et invoquait les morts. Ce karanga ne se fit pas, comme sur un marae, en réponse à celui du groupe hôte et ne reçut aucune réponse en retour. Cependant, comme dans les cérémonies se déroulant sur un marae en pays māori, les diverses unités rituelles se chevauchaient pendant la cérémonie de rapatriement. Pendant que résonnait toujours le karanga, le kaitātaki (challenger), un jeune et vif guerrier, s'est approché pour le wero (défi ou challenge rituel). Il défiait ses hôtes, en courant et grimaçant. Il était torse nu, vêtu d'un piupiu, portait un moko (tatouage) au visage et était armé de son taiaha (arme longue en bois sculpté, bâton de combat) qu'il faisait virevolter de façon menaçante. Il était suivi un peu plus loin dans le cortège par un deuxième jeune homme, qui portait un patu (casse-tête, fort probablement en bois dans ce cas) et vêtu, pour sa part, d'un costume et d'une cravate. Encore ici, un changement a été opéré : le wero est «normalement» exécuté par le groupe hôte. Anciennement, le but était d'effrayer les visiteurs si leurs intentions n'étaient pas pacifiques.

À l'image des rituels de rencontre sur les marae, à l'exception du challenger qui est toujours un jeune homme, les principaux acteurs étaient des personnes plus avancées en âge et les références étaient nombreuses au passé, au $P \bar{o}$ (le monde de la nuit, l'autre monde, celui des dieux et des esprits) et aux ancêtres. Comme l'indique Salmond à propos des rituels de rencontre māori,

«There is a great emphasis on the past, on the ancestors, and on the world of the dead in the rituals, and in this context the old people are most at home. The old people, too, are more tapu than the young. They have had time to acquire esoteric knowledge and to become expert in the rituals, and with old age they draw nearer to the shadowy world of legend, the long night of the underworld or Pō. » (1975 : 120)

Vint ensuite une waiata tangi (chanson de deuil, de lamentation lors des enterrements) exécutée sous la direction des femmes qui étaient au devant de la «procession" et portaient des feuillages autour de la tête auxquelles répondaient le groupe en chœur. Quatre de ces femmes māori se dirigèrent en gémissant et en pleurant au centre de la scène, là où on devinait la présence des vingt toi moko. Les toi moko reposaient dans des caisses en bois recouvertes de deux

à changer puisque plusieurs hommes qui atteignent de nos jours l'âge correspondant au statut n'ont pas une connaissance suffisante de la langue et des rituels. D'autres, plus jeunes, mais possédant davantage de connaissances, les remplacent donc parfois. Le préalable de l'âge tend également à être levé pour les personnes occupant des fonctions importantes dans la communauté (Salmond, 1975 : 126). Dans l'esprit français, d'autres dignitaires avaient pourtant de façon évidente la priorité sur lui.

23. Les mots soulignés sont récités en chœur par le groupe, alors que les mots non soulignés sont prononcés par le leader dans cette cérémonie, par Derek Lardelli. Je fournis ici une traduction française, en remplacement de la traduction anglaise fournie par Salmond (1975: 142). 
korowai, deux capes dont se drapaient traditionnellement les hommes et femmes pour se protéger - autant physiquement que spirituellement -, mais aussi, dans certains cas, en signe de leur statut élevé. Les korowai-qu'ils soient anciens et transmis de générations en générations ou nouvellement fabriqués - servent encore de nos jours à l'occasion de cérémonies importantes comme les mariages, les anniversaires de naissance importants, les cérémonies de remise des diplômes ainsi que pendant d'autres événements formels. Ils symbolisent la fierté culturelle, le prestige, l'honneur et les connexions ancestrales. Ils servent également à couvrir le tūpāpaku (dépouille) et/ou à draper les cercueils lors de tangihanga (funérailles).

" The ultimate honour for departed loved ones is to cover their casket with a cloak, expressing mana [statut, autorité, pouvoir spirituel], aroha [amour inconditionnel] and manaakitanga, or care, and to dress them symbolically for their final journey. » (Tamarapa, 2011 : 11)

En fait, pour ceux qui comprenaient la langue māori, à travers les chants et les incantations, il était évident que les têtes momifiées des ancêtres reposaient sur la scène et que ces ancêtres étaient présents à la cérémonie et les écoutaient (voir également Salmond, 1975 : 167 ; Sinclair, 1990 : 228). C'est d'ailleurs d'abord à eux qu'ils se sont adressés au présent de l'impératif avant de saluer les vivants.

Tel que mentionné précédemment, Salmond (1975 : 179) identifient plusieurs types de hui en fonction de leurs activités centrales. On peut dire que la cérémonie du 23 janvier 2012 comportait deux activités centrales : une visant à souligner une étape particulière du cycle de la vie - les funérailles - pendant laquelle l'attention était portée à des individus particuliers, et l'autre, de nature collective, qui était la signature d'une entente de rapatriement. À ma connaissance, on n'a jamais explicitement évoqué le premier take (objet, motif de la rencontre) dans les communiqués de presse, lors de l'événement comme dans sa couverture médiatique. À la suite de la cérémonie de rapatriement qui s'est tenue à Rouen en mai 2011, sous la direction d'un autre kaumatua (aîné) ${ }^{24}$, on pouvait pourtant lire dans le New Zealand Herald la chose suivante à propos de cette cérémonie :

«Before the ceremony started, Te Herekiekie Herewini, Te Papa's repatriation manager, explained that the ceremony would be like a tangi [forme abrégée de tangihanga, funérailles] and during the formalities a korowai (Māori cloak) was placed over the head, simi- lar to a practice on marae, which keeps bodies warm during mourning periods. » (Tahana, 2011)

Le directeur des rapatriements fait donc une allusion directe à des funérailles. Il est d'ailleurs intéressant de se rapporter à un texte de Dansey (1975), lequel me porte à penser que cette première cérémonie est vue comme une première étape ou un moment de la cérémonie de deuil de ces guerriers dont les têtes reposent à l'étranger depuis de nombreuses décennies. Ainsi, parlant de la mort au combat à l'étranger de soldats māori, entre autres, lors des deux guerres mondiales, Dansey identifie plusieurs étapes au deuil :

"First there is the mourning on the battlefield, usually brief and poignant, the burial according to military custom and with Christian ritual.

Secondly, in the battlefield situation, there is sometimes the vow to obtain utu [vengeance, réparation] for the fallen.

Thirdly, there is the mourning at home, the tangihanga in nearly all its parts, except that the body is not there.

Fourthly, at the end of the war-or at convenient times during the war - there are visits to battlefield and cemeteries where the soldiers take part in services and farewell their comrades before returning home. [...]

Fifthly, there is the return home and the tangihanga on many maraes.

Sixthly, there is the erection of memorials, gates, stones, flagpoles, houses, in memory of the dead.

Seventh is the commemoration in song, this often being done during the war itself.

These songs go on and on and are a living, most important part of present-day Māori life. » (1975: 186-187)

Dans le cas qui nous intéresse, la cérémonie de rapatriement serait donc un de ces moments. On peut supposer qu'elle succède à une première phase de la cérémonie du deuil qui se tint sur le champ de bataille, peu après la mort de ces guerriers, s'ils furent chanceux :

«[s]ometimes when an action was fought near home there would be a tangihanga over the dead, the women coming on to the battlefield searching for their own close relations. » (Dansey, 1975 :184)

$\mathrm{Au}$ retour en Nouvelle-Zélande, le 27 janvier 2012, une cérémonie d'accueil pour souligner le retour des ancêtres au pays suivit celle ayant eu lieu au musée du quai Branly. Elle se tint au marae du musée Te Papa en présence de plusieurs leaders tribaux, des officiels du musée et du gouvernement et de l'Ambassadeur de la France en Nouvelle-Zélande, Francis Etienne. Les toi moko firent leur entrée sur le marae dans les caisses de

24. Dans ce cas, le kaumatua était Te Kanawa Pitiroi, un autre membre du groupe d'experts sur le rapatriement au Te Papa. Le leader donne habituellement sa couleur - et celle de sa tribu - à la cérémonie. 
bois portées, entre autres, par des membres de l'équipe de rapatriement composant un cortège funèbre, accueilli dans l'esprit de la coutume par un groupe de pleureuses, des femmes endeuillées appelées pani (Sinclair, 1990 : 228), qui gémissaient et pleuraient à leur arrivée (Ngata, 2005 : $33)^{25}$. Dans le monde māori :

« [o]pen grieving and outpouring of emotion is encouraged ( (Higgins et Meredith, 2011). « Tears should be unrestrained and mingle freely with those of the bereaved. All of this is evendence of aroha, of emphaty, sympathy, heart, and sadness and is viewed as a major distinguishing feature of Mãori. » (Sinclair, $1990: 229$; voir également Dansey, 1975)

Le groupe de femmes se sont par la suite assises tout autour des toi moko après qu'on les ait déposés sur le porche de la wharenui (maison de rassemblement) et qu'on les ait recouverts de kākahu. Pou Temara, président du groupe d'experts sur le rapatriement au Te Papa, qui fut un des orateurs qui prit la parole pendant la période du whaikōrero (échanges oratoires), eut ces mots à l'attention des ancêtres dont les dépouilles rentraient au pays :

"You've been away from New Zealand for over 200 years. Welcome back. Now your sleep will be a restful one, will be a peaceful one. » (in Schwartz, 2012)

Une autre cérémonie encore aura lieu lorsque les toi moko dont les recherches permettront de retracer les origines seront rendus à leur tribu en vue de leur enterrement dans leur lieu final de repos sur la terre de leurs ancêtres. Dans le monde māori, ces cérémonies sont essentielles :

" There is a need for ceremonial so that the dead may be properly farewelled, his or her virtues extolled (and, quite often, faults and failings almost brutally enumerated), the bereaved comforted, the ties of relationship renewed and the deep well of Maoritanga [Māori culture, domaine ou univers de sens māori] tapped so that all who come may refresh and strengthen themselves. " (Dansey, 1975 : 180)

Pour en revenir à la cérémonie qui eut lieu au musée du quai Branly, les quatre femmes qui marchaient au devant de la procession et qui portaient des feuillages sur leur tête s'agenouillèrent et s'assirent à même le sol, deux de chaque côté des toi moko. Elles restèrent près d'eux tout au long de la cérémonie et continuèrent à leur tenir compagnie, même lorsqu'elle prit fin pour d'autres karakia (prières) à l'abri du regard du public. Des membres de l'équipe responsable du rapatriement versés en tikanga (tradition), karakia (prière) et kawa (protocole) ont d'ailleurs raccompagné les toi moko pendant tout leur voyage de retour en Nouvelle-Zélande en avion (Te Papa, s.d.). Encore cette fois, comme l'indiquait en mai 2011 la porte-parole de Te Papa, Anna Wilson, à la suite de la cérémonie de Rouen, les toi moko furent « handled the same as dead bodies » (Hunt, 2011). Fut donc suivie la pratique habituelle māori qui est à l'effet de ne jamais laisser seul le tūpāpaku (dépouille) jusqu'à son enterrement puisque le wairua (esprit) de la personne décédée est sensé l'accompagner pendant un certain temps et il convient de lui tenir compagnie (Sinclair, 1990 : 228 ; Salmond, 1975 : 182 ; Higgins et Meredith, 2011). C'est aussi la raison pour laquelle on entoure habituellement la dépouille de portraits de famille, incluant des photos d'ancêtres décédés, et de taonga de famille tels que des pendentifs en jade afin de réaffirmer les liens familiaux et la continuité entre les vivants et les morts. On pouvait d'ailleurs remarquer sur la scène du théâtre Claude Lévi-Strauss plusieurs portraits de personnes décédées et deux d'entre eux encadraient les toi moko.

"Our dead are very close to us in Maoridom. They do not lie alone in that short space between death and burial. We stay with them every minute and talk to them and sing to them. When we have returned them to the earth we remember them in song and speech. Each time we meet one another after being apart we pause and weep again, no matter how happy the occasion for our meeting. » (Dansey, 1975 : 188)

"[T]he deceased must be kept constantly warm and comfortable by the presence of kinfolk, in order to calm the soul and assist it on its journey to the spirit world. » (Ngata, $2005: 33$ )

Sinclair (1990 : 229) mentionne que le rituel des funérailles accorde une place très importante aux femmes, car ce sont elles qui veillent sur la personne décédée. Ceci fait d'elles des gardiennes de la Maoritude et des façons de faire héritées des ancêtres puisque le tangihanga est vu comme fournissant un ancrage indispensable dans la tradition, un site d'affirmation de l'appartenance à une collectivité qui inclut les ancêtres et donc de la continuité entre le passé et le présent, ainsi qu'un site politique important permettant aux Māori d'insister sur leur statut particulier au sein de la société néo-zélandaise (voir aussi McIntosh, 2001 ; Oppenheim, 1973). Il est d'ailleurs intéressant de souligner que :

" the tangi [...] is revered as the most "Māori" gathering of all [...] The tangi takes precedence over all other hui, and indeed over all other activities. » (Salmond, 1975 : 180) 


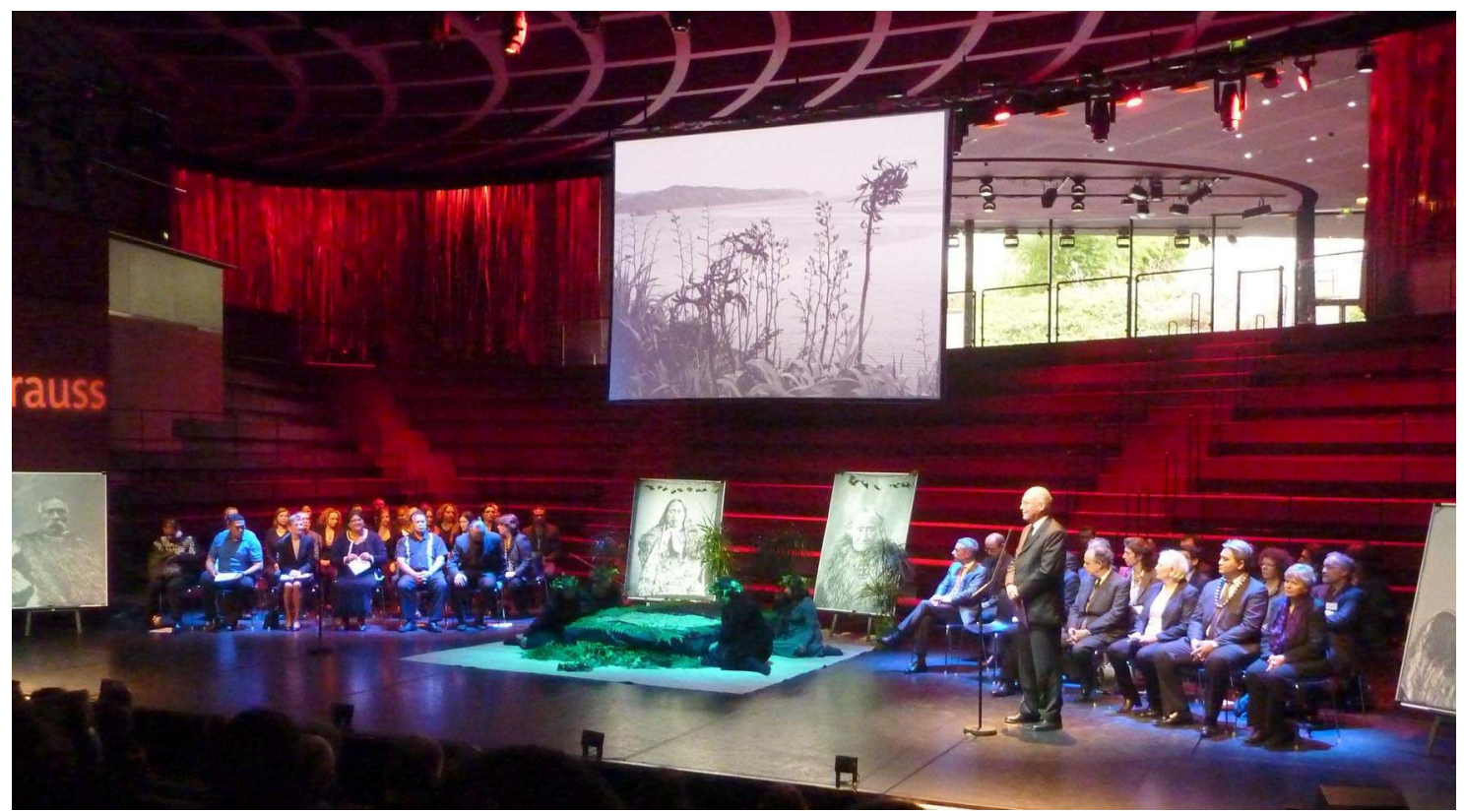

Рното 1. - Körero (discours) du président du musée du quai Branly. Il faut remarquer la disposition des participants à la cérémonie ainsi que des toi moko au centre de la scène, entourés de pleureuses et de photos de Māori maintenant décédés (cliché Natacha Gagné)

C'est dire l'importance que les Māori accordent à ce type de rituel qui a d'ailleurs beaucoup gardé de sa forme traditionnelle en faisant peu de concessions aux pressions modernes (Sinclair, 1990: 231). C'est dire également toute l'importance accordée à cette cérémonie de rapatriement.

Les autres membres du groupe des visiteurs se joignirent au tangi en baissant la tête et, pour certains, en versant quelques larmes. À leur arrivée sur la scène, ils firent quelques pas en direction des toi moko en regardant dans leur direction avant de prendre place à gauche de la scène, se disposant sur trois rangées, faisant face à leurs hôtes. Avant de rejoindre sa place, Michelle Hippolite laissa tomber les feuillages qu'elle avait sur la tête au chevet des toi moko, laissant ainsi une part d'elle près d'eux, comme le font les visiteurs aux pieds de la personne décédée lors d'un tangihanga (Salmond, 1975: 184). Les hommes māori prirent place sur la première rangée, comme le veut la coutume. Deux femmes, Michelle Hippolite et l'Ambassadeur Rosemary Banks, firent aussi de même, ce qui contrevenait à l'habituelle façon de faire sur un marae. Selon les protocoles «traditionnels», lors des échanges cérémoniels sur les marae (ou ailleurs), les hommes s'assoient sur la première rangée, suivant un ordre particulier: les plus âgés et ceux ayant plus de mana (pouvoir spirituel, autorité, statut, prestige) ayant la priorité sur les autres.
Viennent ensuite, sur une (des) rangée(s) derrière celle(s) des hommes, les femmes suivant une même priorité d'âge et de $m a n a^{26}$.

Pour l'étape suivante du rituel, le whaikōrero (échanges oratoires), le président du musée du quai Branly, Stéphane Martin, principal représentant du groupe hôte puisque la cérémonie prenait place dans «son » établissement, prit la parole en premier, comme c'est l'usage. Il prononça son discours entièrement en français. Une traduction simultanée était offerte aux membres de la délégation néo-zélandaise. Elle s'adressait en priorité à ceux assis dans la première rangée, en particulier aux hommes. Stéphane Martin invita à la fin de son intervention le kaumatua Derek Lardelli à prendre la parole.

Dans un style qui reprend pour l'essentiel le style général et la structure des kōrero (pour des détails, voir Salmond, 1975 : 158-176 ; voir également le schéma ci-dessus), il a d'abord récité sous la forme d'une incantation rapide et rythmique un tauparapara, un chant aux mots anciens et aux références ésotériques. Cette incantation était illustrée par les gestes vigoureux de l'orateur. La partie qui suivit, toujours fort stylisée, laissait davantage de place à l'improvisation tout en étant toujours située dans un "paysage mythologique » (Salmond, 1975 : 165). Il s'adressa d'abord aux ancêtres. L'orateur aborda ensuite le sujet spécifique de la rencontre: le rapatriement des toi moko et 


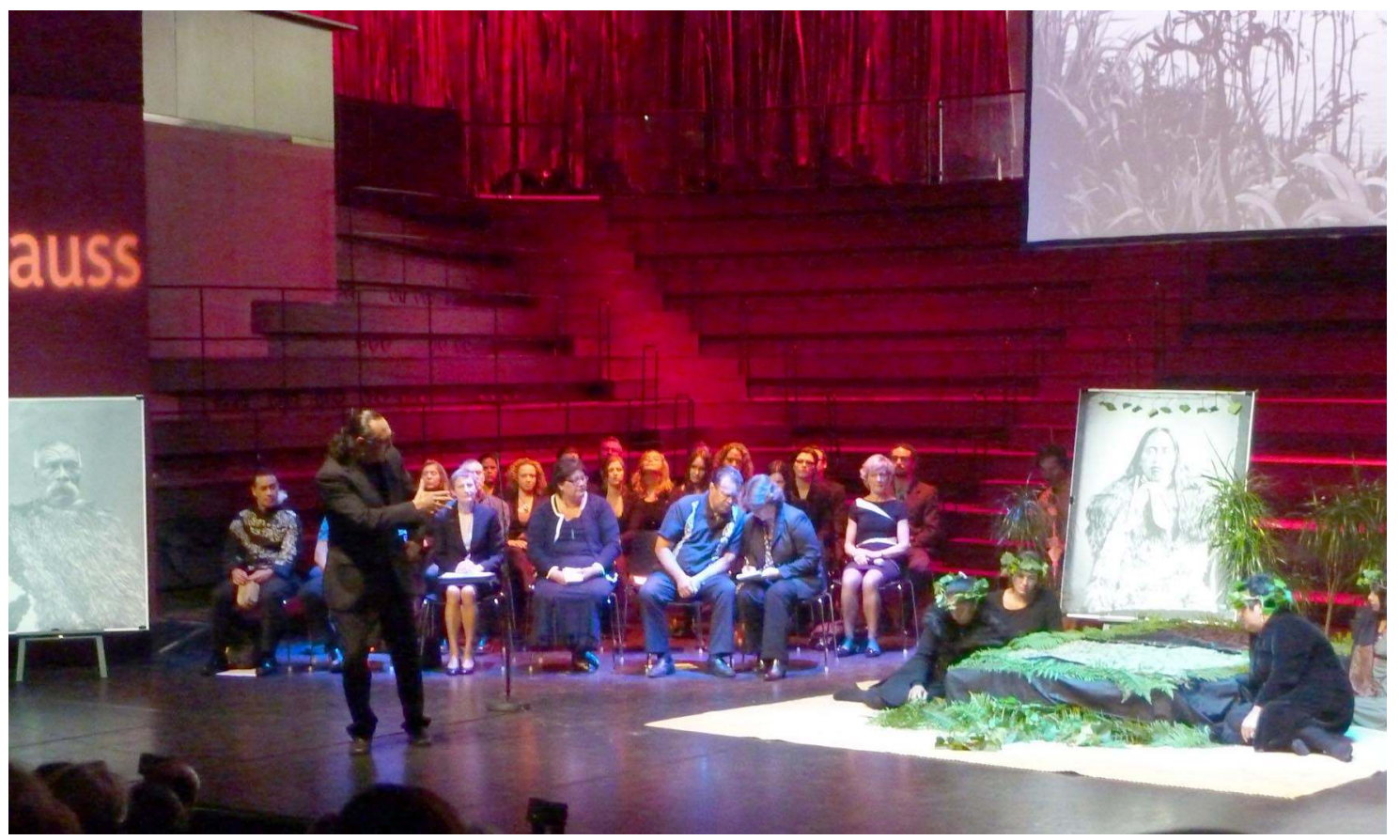

Рното 2. - Kōrero (discours) du kaumatua Derek Lardelli. On le voit ici s'adresser aux ancêtres dont les têtes reposent sous les korowai (cliché Natacha Gagné)

s'adressa directement à ses vis-à-vis français pour les remercier. Il fit son intervention complètement en Māori. Une traduction sera fournie plus tard, après la waiata (chanson traditionnelle) en appui à sa prestation. Tauroa and Tauroa (1986: 72) insistent sur le fait qu'en Nouvelle-Zélande, si l'anglais peut être utilisé dans toutes les autres occasions, il est en général très important pour les Māori que leur propre langue soit utilisée durant les rituels d'accueil se passant sur la cour cérémoniel et en particulier pour le whaikōrero. Extrêmement peu de compromis sont faits sur cette question linguistique.

Par sa performance, Derek Lardelli a sans contredit impressionné ses hôtes même s'ils n'ont compris que plus tard ce qu'il disait. Sans que je sois une spécialiste en la matière, il m'a semblé faire preuve par sa gestuelle et son aisance d'un talent qui pourrait un jour, quand il sera plus vieux, lui permettre de rivaliser avec les meilleurs orateurs :

"The best speakers [...] move freely on the marae, striding backwards and forwards, leaping on their turns (pekepeke), quickly stamping their feet and lunging into powerful gestures. They more like warriors, agile and strong. » (Salmond, 1975 : 172)

Lardelli finit sa prestation en déposant par terre, près des hôtes, douze koha. Il s'agissait de petits kete (petit sacs tissés de fibres de lin) contenant des pendentifs en jade (pounamu), une pierre que les Māori considèrent comme un taonga (trésor) parce qu'elle possède un grand mana (statut, autorité, pouvoir spirituel), tant au plan spirituel que matériel. Plusieurs des membres de la délégation néo-zélandaise en portaient également.

«Pounamu plays a powerful role in affirming and building many types of relationship - from the personal to the political. [...]

In the past, families exchanged pounamu treasures at marriages to symbolise their new connection. Former enemies presented each other with gifts, such as mere pounamu (nephrite weapons), to establish lasting peace. In some districts, this making of peace was called te tatau pounamu - a 'greenstone door', that is, a door to close off the deeds of the past.

Today, pounamu continues to be a meaningful gift. It is sometimes exchanged between the government and iwi (tribes) to honour important agreements. 》 (Museum of New Zealand Te Papa Tongarewa, s.d.)

Ce cadeau tombe donc dans les façons de faire traditionnelles et correspond tout à fait aux objectifs à l'origine de la rencontre. Après avoir déposé les douze kete contenant les pendentifs sur le sol, Lardelli en accrocha ensuite un au cou du ministre de la Culture, Frédéric Mitterrand, lui fit le hongi, et invita de la main les autres membres du groupe hôte à venir chercher un des koha déposés sur le sol.

Les membres de la délégation française parurent à ce moment un peu déconcertés puisque tout se passait en māori et il m'a semblé y avoir 


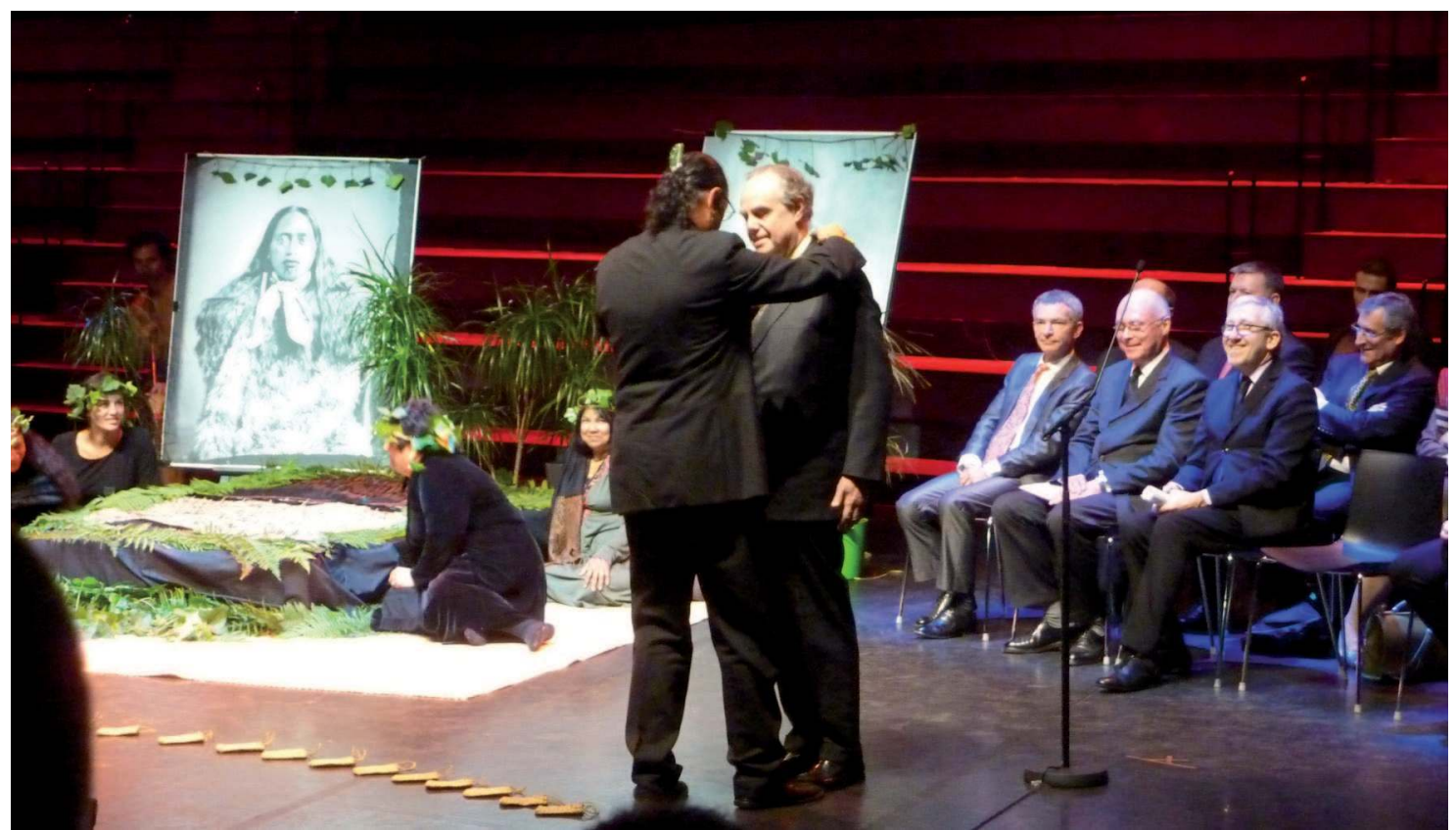

Рното 3. - Le kaumatua Derek Lardelli offre un роипати au ministre Frédéric Mitterrand (cliché Natacha Gagné)

un moment d'hésitation. Stéphane Martin se leva en premier et d'autres membres du groupe hôte le suivirent et prirent un koha sur le sol. La prestation de Derek Lardelli fut suivie comme il se doit par une waiata (chanson traditionnelle). Celle-ci fut exécutée sous la direction de la femme māori la plus âgée parmi celles agenouillées près des toi moko. Il est intéressant de noter qu'au moment où les femmes ont commencé à chanter, accompagnées par le groupe des visiteurs, l'assistance s'est levée de façon spontanée. Elle se lèvera de la sorte au son de la waiata qui suivra chacune des interventions des membres de la délégation néo-zélandaise. En contexte māori, seuls les membres du groupe de l'orateur se lèvent pour leur montrer leur soutien. Je n'ai pas pu m'empêcher de voir ici des traces du rituel catholique. On peut penser que les Māori ont bien réussi à transmettre le caractère sacré de la cérémonie et à transporter ainsi les Français présents dans l'assistance et sur la scène dans une atmosphère empreinte de religiosité.

Le don du koha clôt habituellement le moment du rituel consacré aux échanges oratoires et le hongi clôt habituellement la partie cérémonielle, tapu (sacrée), d'un hui (rencontre). Dans la cérémonie du 23 janvier 2012, un hongi et un échange de poignées de mains plus généralisé auront lieu plus tard, après la signature de l'entente de rapatriement à la toute fin de la cérémonie de rapatriement. Cependant, il apparaît raisonnable de dire que le hongi entre Derek Lardelli et le ministre de la Culture permit une levée du tapu qui fit entrer les participants dans la deuxième phase de la rencontre davantage centrée sur le take (objet, motif) principal : l'entente de rapatriement des toi moko.

Après un résumé en français des principaux éléments évoqués par Derek Lardelli dans son discours, d'autres intervenants - dont trois femmes $^{27}$ - se levèrent et prirent la parole dans l'ordre suivant: Madame l'ambassadeur de Nouvelle-Zélande, qui dira quelques mots de salutations en māori avant de passer au français; Madame Hippolite, kaihautū (leader māori) dans l'équipe de direction du Te Papa ; Madame la sénatrice de la Seine-Maritime; et Monsieur le ministre de la Culture. Les deux interventions des Néo-Zélandaises furent suivies par des waiata (chanson traditionnelle). Quant à Michelle Hippolite, elle ouvrit son intervention par un chant de lamentation qui revêtait une importance symbolique puisqu'elle expliqua qu'il fut écrit lors de la tournée de l'exposition Te Maori dans les années 1980. Pendant son allocu-

27. Selon l'interprétation que je suggère, même si elles furent assises sur la première rangée, ces trois femmes ne contrevinrent pas fondamentalement aux règles générales relatives aux genres, puisqu'elles prirent la parole dans la partie noa (libre de toute restriction religieuse, ordinaire, détendue; complément et antidote au tapu) de la rencontre pendant laquelle les femmes, comme les plus jeunes, peuvent prendre la parole de façon beaucoup plus libre. 


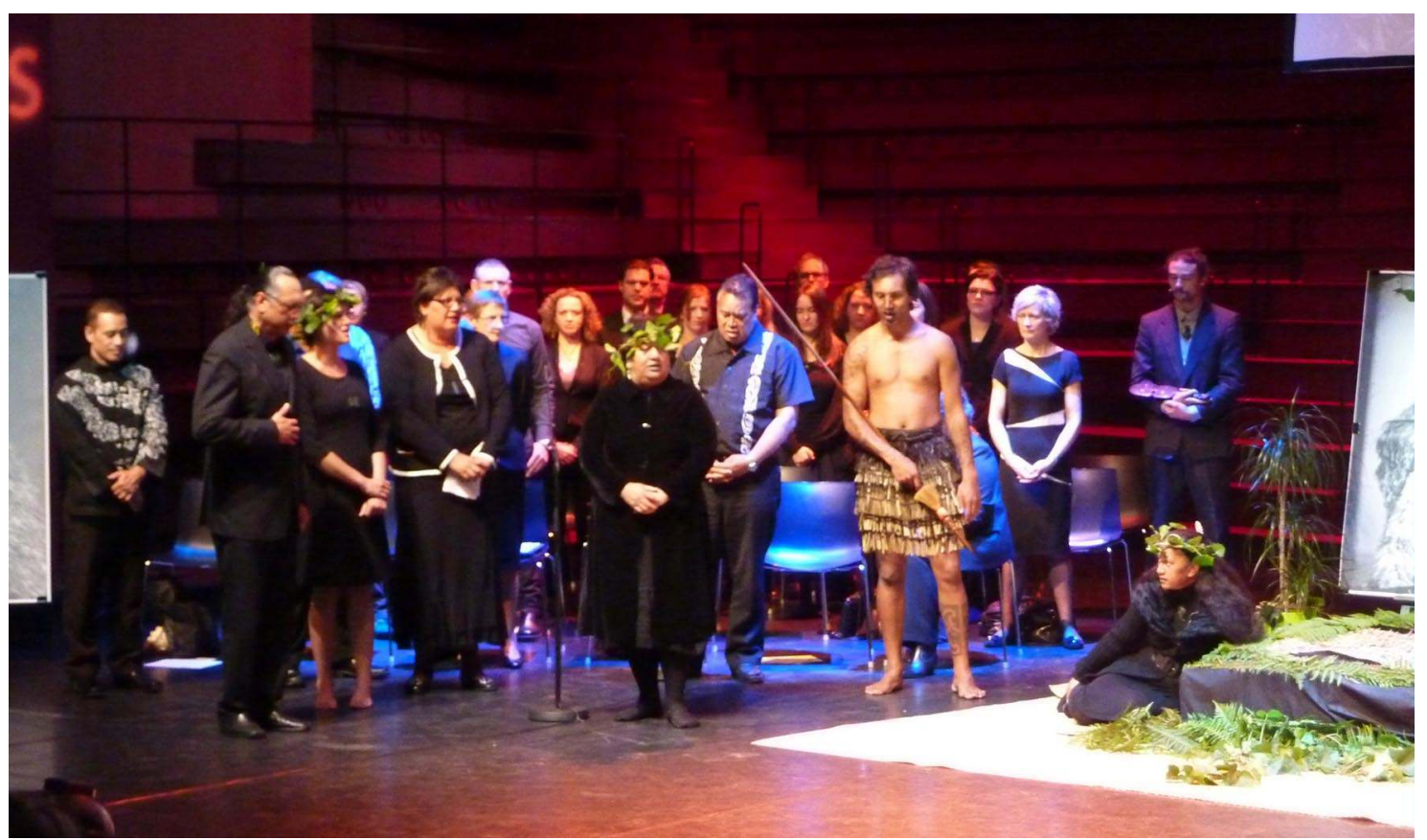

Рното 4. - Waiata sous la direction de la femme māori la plus âgée, accompagnée par l'ensemble du groupe en chœur. Il faut remarquer la présence du kaitātaki (challenger) sur sa droite (cliché Natacha Gagné)

tion, en anglais, mais ouverte par quelques phrases en māori et bénéficiant de la traduction d'une interprète au fur et à mesure de sa présentation, un flot d'émotions la contraint quelques instants au silence. Les hommes māori du groupe se rapprochèrent d'elle à ce moment, en signe d'appui.

L'intervention du ministre Mitterrand fut suivie par la signature de l'accord formalisant les conditions de la restitution et l'échange de hongi et de poignées de mains. Stéphane Martin fournit quelques explications quant au contenu de l'entente et, après un applaudissement de la sénatrice à la demande du ministre Mitterrand, invita l'assistance à prendre un verre dans l'espace bar attenante au théâtre. En contexte māori, c'est bien à l'hôte de clore la rencontre cérémonielle et d'inviter les invités à partager un goûter, mais il m'a semblé lire la surprise sur les visages des représentants de la délégation néozélandaise à ce moment, surprise que j'ai interprétée comme étant liée à ce qui m'est apparu être une clôture un peu précipitée. Le groupe de visiteurs s'est alors rapidement regroupé sur la scène pour faire, me semble-t-il, une karakia (prière) pour clore le hui. Les rencontres sur les marae se terminent habituellement par l'échange de quelques remerciements, de chants en guise d'au revoir et de karakia avant que les visiteurs sortent de l'enceinte du marae et reprennent leur route.

\section{Inversion symbolique et affirmation politique}

Voyons maintenant comment se compare la cérémonie de rapatriement avec un rituel de rencontre type. À partir de l'ouvrage de Salmond (1975), j'ai synthétisé dans un premier tableau (figure 2) les différents moments ou unités de la séquence rituelle d'une cérémonie de rencontre « traditionnelle» se tenant sur un marae. J'ai divisé le tableau en deux afin d'indiquer les unités auxquelles participaient chacun des groupes impliqués. J'ai également inséré des indications quant au sexe des principaux protagonistes associés à chaque moment ou unité.

Dans un deuxième tableau (figure 3), j'ai tenté une synthèse des principaux moments de la cérémonie de rapatriement du 23 janvier 2012 afin de faciliter aux lecteurs la comparaison.

Bien que prenant place à l'étranger, bien loin des marae - les lieux traditionnels de rassemblement māori et centres cérémoniels - dans une institution occidentale, le musée ${ }^{28}$, longtemps associée à l'entreprise coloniale, la cérémonie avait une signature bien māori. On constate que la cérémonie de rapatriement comprenait toutes les unités rituelles identifiées par Salmond (1975) comme constituant le rituel de rencontre, même si des aménagements ont été faits afin de parer à l' « incompétence » des hôtes en matière de rituels māori. Ainsi, l’ordre de la séquence a été

28. Je ne m'étendrai pas ici sur le sujet, mais le musée du quai Branly, institution hôte de la cérémonie de rapatriement analysée ici fut au cœur des débats sur le rapport à l'Autre et la situation postcoloniale ainsi que sur la mission civique et politique des musées (voir, parmi de nombreux exemples, De l'Estoile, 2007 ; Jolly, 2011 ; Price, 2011). 


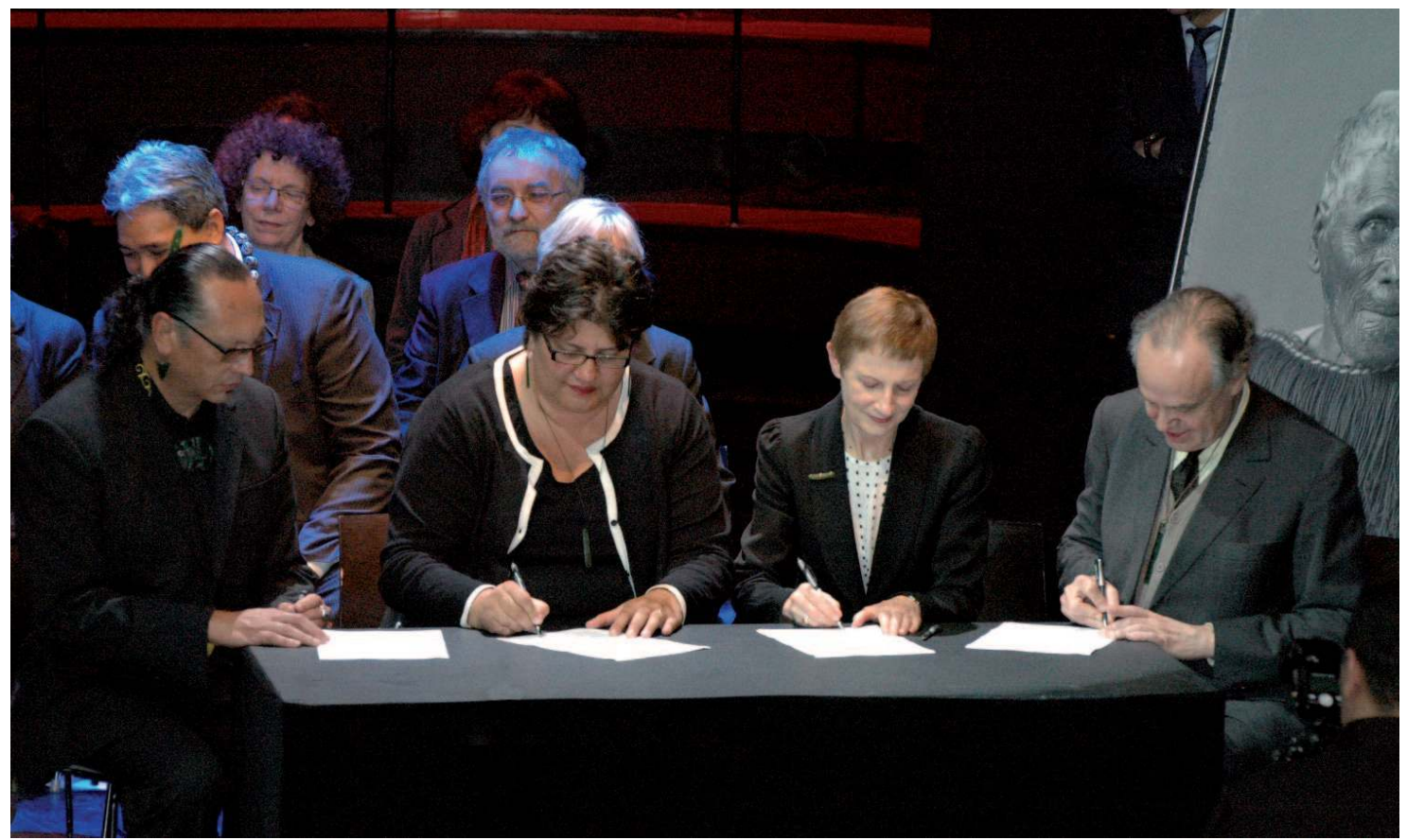

Рното 5. - Signature de l'entente de rapatriement par Derek Lardelli, Michelle Hippolite, Rosemary Banks et Frédéric Mitterrand (cliché Isabelle Leblic)

légèrement modifié et les visiteurs ont pris sur eux la responsabilité de toutes les unités rituelles en amont du whaikōrero. D'autres solutions auraient pu être possibles. Par exemple, ils auraient pu " prêter » une kai karanga (femme qui exécute l'appel cérémoniel de bienvenue) et un kaumatua (aîné) aux hôtes afin qu'ils puissent assurer leur part des responsabilités rituelles, ce qui se fait souvent en NouvelleZélande quand un groupe de visiteurs non māori, par exemple, est accueilli sur un marae. Cette option, pour le groupe de visiteurs, a probablement été écartée puisqu'elle aurait poussé trop loin l'imposition de son propre kawa (protocole), alors que l'occasion commandait une certaine humilité - une valeur chère aux Māori et d'être conciliants devant cette ouverture et ce geste de réconciliation de l'État français.

La cérémonie s'est trouvée malgré tout à opérer une inversion des rapports qui déterminent habituellement le format des rituels de rencontre : alors que ce sont habituellement les hunga käinga (gens du lieu, hôtes) qui déterminent le kawa (protocole) et les tikanga (traditions) qui orienteront le rituel, les Français furent plutôt transportés dans l'univers māori, opérant une inversion symbolique qui traduit également, à mon sens, un renversement du rapport politique en faveur des Māori.

Comme l'indique Salmond (1975 : 12), Metge (1995) et mes propres travaux parmi les Māori vivant en ville (par exemple, Gagné, 2011, 2013), la société māori s'articulent autour de quelques grands principes. Dans le contexte de rencontres cérémonielles, les principaux principes à l'œuvre, selon Salmond (1975), sont ceux de mana (statut, autorité, pouvoir spiritual), tapu (sacré ou pollué, polluant, sous restriction) et utu (retour pour quelque chose reçu, que ce soit en bien ou mal; réciprocité, compensation). L'inversion symbolique qui s'est produite à travers la cérémonie de rapatriement a donc permis aux Māori d'affirmer leur mana en donnant à voir leur assurance, leur maîtrise du rituel, une culture encore bien vivante, des performances oratoires impressionnantes qui n'ont rien à envier à celles des hôtes français. Ils ont su créer une atmosphère solennelle empreinte de tapu, une sacralité qui était facilement décodable, même pour les noninitiés, comme nous l'avons vu. Ils ont également réussi à faire passer l'assistance ainsi que leurs hôtes par une gamme d'émotions : l'intensité du pōwhiri (chants mimés d'accueil) et les impressionnantes performances du kaitätaki (challenger) et du kai kōrero (orateur ; dans ce cas, Derek Lardelli) ont fait frissonner l'assistance qui a été également émue par les pleurs des femmes agenouillées à côté des dépouilles mortuaires et charmée par les chants.

Du point de vue des Māori, dans un contexte de décolonisation, cette cérémonie de rapatriement - comme celles qui accompagnèrent les 


\begin{tabular}{|c|c|c|}
\hline \multirow{7}{*}{ 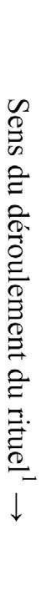 } & Hunga kāinga & Manuhiri \\
\hline & & Waerea ô \\
\hline & Wero ô & \\
\hline & $\begin{array}{c}\begin{array}{c}\text { Karanga } \text { 早 } \\
\text { (commence) }\end{array} \\
\end{array}$ & $\begin{array}{c}\text { Karanga } \text { q } \\
\text { (répond) }\end{array}$ \\
\hline & Pōwhiri & \\
\hline & Tangi 우 & $\begin{array}{c}\text { Tangi } \text { 早 } \\
\text { (commence) }\end{array}$ \\
\hline & $\begin{array}{c}\text { Whaikōrero } \hat{\delta} \\
\text { (commence, puis ensuite alternance entre } \\
\text { locaux et visiteurs ou tous les locaux et } \\
\text { tous les visiteurs) }\end{array}$ & $\begin{array}{c}\text { Whaikōrero } 0 \\
\text { (répond) }\end{array}$ \\
\hline
\end{tabular}

FIGURE 2. - Structure d'un rituel de rencontre type ${ }^{29}$

\begin{tabular}{|c|c|c|}
\hline \multirow{8}{*}{ 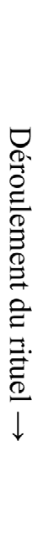 } & Hunga kāinga & Manuhiri \\
\hline & & Waerea $\widehat{0}$ \\
\hline & & Pōwhiri \\
\hline & & Karanga ㅇ \\
\hline & & Wero ठ̊ \\
\hline & & Tangi 웅 \\
\hline & $\begin{array}{c}\text { Whaikōrero ô } \\
\text { (commence) }\end{array}$ & $\begin{array}{c}\text { Whaikōrero ô } \\
\text { (répond) }\end{array}$ \\
\hline & & \\
\hline
\end{tabular}

FIGURE 3. - Structure de la cérémonie de rapatriement du 23 janvier 2012

rapatriements précédents - m'apparaît également être une façon de mettre en œuvre le principe de utu: c'était l'occasion de prendre une revanche, de rétablir le mana, en renouant les liens avec les ancêtres dont les têtes avaient fait l'objet d'une transaction et en soulignant leur grandeur, mais plus largement en affirmant l'autorité et le désir d'autodétermination de tout un peuple après près de 200 ans de minorisation. Dans la cérémonie du 23 janvier 2012, une grande importance était accordée aux ancêtres et au passé, celui mythique des Māori, mais également celui de la conquête coloniale et du com- merce des toi moko et autres objets māori (comme des armes, des ornements, des outils, des vêtements et des sculptures) dans le contexte de "l'activité d'inventaire ethnologique du monde » (De L'Estoile, $2007: 137)^{30}$.

La symbolique du tangihanga (funérailles) rendait d'autant plus forte l'affirmation de leur autorité par les Māori, tant par son côté dramatique que par l'accent qui y est mis sur la continuité avec le passé :

« Tangi make clear assertions that authority is still to be found in ancestral traditions. [...] The emphasis on continuity with the past is an intense refusal to relinquish

29. Selon les tribus et les régions, l'ordre peut être légèrement différent. Certaines unités rituelles peuvent également se chevaucher à l'exception, selon mon expérience, du whaikōrero qui bénéficie de la pleine attention de tous. Salmond (1975: 147) rappelle également qu'on réfère à cette portion du rituel par le terme mihimihi d'où proviendrait le terme mihi utilisé pour parler de l'ensemble de la cérémonie d'accueil, ce qui indiquerait l'importance et la centralité du whaikörero. De nos jours, le terme pōwhiri est aussi souvent utilisé pour désigner l'ensemble de la cérémonie d'accueil (voir, par exemple, Matenga-Kohu et Roberts, 2006).

30. Voir McCarthy (2007) pour une histoire des objets et autres aspects de la culture māori prisés par les Européens et l'histoire de leur mise en exposition de la rencontre coloniale jusqu'à aujourd'hui. Le livre fait également état de la résistance et de la participation māori à la mise en exposition de leur culture, puis de leur contrôle sur celle-ci. 
their heritage and become part of the Pakeha world. By looking to their ancestors, to their past, they reaffirm the value of being a Mãori in the present. » (Sinclair, 1990 : 233)

Comme le souligne encore Sinclair, "[t]he tangi provides an arena in which Māori may proclaim their autonomy and unity» (1990: 232). C'est une occasion pour redéfinir leur position dans la société néo-zélandaise, mais également dans le monde. Cette cérémonie ainsi que les processus de rapatriement des dernières années sont, pour les Māori, une façon d'affirmer leur autorité face aux Néo-Zélandais non māori et d'agir en partenaire vis-à-vis l'État néozélandais et de ses institutions, y compris à l'international. À cet effet, le fait que la cérémonie soulignait le retour de toi moko aux leurs était particulièrement significatif :

"In the past this type of gesture was seen as a "tatau pounamu" or a "doorway to welcomepeace", through the return of a captured Toi moko to their respective whänau (family) of origin. The return of an ancestral head provided reconciliation for warring tribes and allowed for a period of peace for the two enemies. The significance of this legislation by the French government is not lost on Màori tribes and communities, and will endure in the Māori memory, as it in turn allows Māori to find resolutionandreconciliation withour own historical inter-tribal conflict within New Zealand. »(Herewini, 2011:1)

Le tangi, dans le cadre néo-zélandais, serait un moment clé pour les Māori pour changer les termes de la relation avec les Pākahā (néozélandais d'origine européenne), y compris en affirmant une supériorité morale.

«The tangi and the values associated with it arm its participants with a moral counterchallenge, for this is an arena that is subject to Mãori, not European, definitions. At a tangi, Māori virtues are implicitly and explicitly opposed to European ways. For once, Europeans come up lacking. Pakeha materialism loses ground to assertions and demonstrations of Māori spirituality. Māori generosity and hospitality emerge as superior values when compared to rigid Pakeha politeness that, in Māori eyes, masks a lack of caring. [...] While Pakeha are seen as motivated by self-interest, the tangi provides a conclusive demonstration that Māori are concerned with their family, their near and remote kin, and with anyone who has a lien, however indirect, on their affections. » (Sinclair, $1990: 232$ )

Lors du 23 janvier 2012, le caractère solennel de la cérémonie créa aussi peut-être et chez certains ce sentiment d'une supériorité morale des Māori, sentiment renforcé par les vertus qu'on attribue aux opprimés et en particulier, aux peuples autochtones, les ONG, les diverses instances de l'ONU ainsi que certains chercheurs n'ayant de cesse de rappeler ces vertus, pêchant même parfois par essentialisme et par une sorte d'utopisme (Niezen, 2010 ; Sissons, 2005). Ce sentiment a pu également être renforcé par le fait que les hôtes parurent à quelques reprises plutôt décontenancés, ne sachant pas toujours comment réagir et quoi faire. Une certaine confusion semblait être présente au moment d'accepter les koha (présents), par exemple. Ce fut également le cas lors $\mathrm{du}$ hongi. Certaines actions et paroles - j'en ai identifié quelques-unes - des Français parurent également déplacées.

De tels moments offrent donc des opportunités pour les différentes parties de réarticuler leurs relations, tant historiques que contemporaines. Tous les orateurs, y compris les non-Māori ont souligné cette volonté d'établir de nouvelles relations dans leur discours. L'exécution du rituel en tant que tel peut également être analysée comme une suite d'actions qui engendrent des changements réels. Comme l'indique Préaud, dans un contexte cérémoniel un peu différent :

« it is not only about politeness or diplomacy, it is about defining by negotiation and making official (actual) through performance a new situation for relationships to make sense. » (2011 : 90-91)

Pourtant, si on se rapporte à nouveau à la structure rituelle de la cérémonie du 23 janvier 2012, on s'aperçoit que des compromis ont été faits par les Māori en vue de réarticuler les relations. Ils furent également nécessaires en vue de la bonne marche de chaque étape de la cérémonie. On peut penser que le succès de la cérémonie était extrêmement important puisque comme dans le cas de n'importe qu'elle hui, « [ $\mathrm{t}]$ he ritual has to be gone through if things are to be "tika" (correct) » (Salmond 1975 : 165). La bonne marche du rituel était sans doute vue comme un élément essentiel pour assurer le retour sûr et sans risque des toi moko vers leurs terres ancestrales.

Enfin, ce cas nous rappelle ce qu'a déjà montré Schwimmer (1972) (voir également Gagné, 2008 : 108-109) : la concurrence entre deux groupes - un minoritaire et l'autre majoritaire - n'est jamais simplement symbolique. Elle est toujours liée à des asymétries tant politiques qu'économiques, et donc aux possibilités de concurrence réelle. La cérémonie fut donc l'occasion de se présenter dans des termes différents face à une ancienne puissance coloniale et ses institutions, dont les musées font partie. Comme le rappelle McCarthy :

«[w]hile the museum was itself a colonial artefact, it was never an unchallenged tool of settler rule and Māori 
always attempted to steer it towards their own ends. 》 (2007: 202)

La cérémonie du 23 janvier 2012 faisait partie de ces tentatives qui m'est apparue comme réussie et porteuse de changements. Elle doit être comprise dans la longue histoire des luttes māori et de leur projet politique. Elle doit également être comprise dans l'actualité postcoloniale de la France, mais je laisserai à d'autres le soin de faire ce travail de contextualisation.

\section{BIBLIOGRAPHIE}

ALLEN Chadwick, 2002. Blood Narrative. Indigenous Identity in American Indian and Maori Literary and Activist Texts, Durham and London, Duke University Press.

BARKer Ian, 2006. The Protection of Cultural Heritage Items in New Zealand, in Barbara T. Hoffman (ed.), Art and Cultural Heritage: Law, Policy \& Practice, Cambridge, Cambridge University Press, pp. 145-147.

Belich James, 1996. Making Peoples: A History of the New Zealanders: From Polynesian Settlement to the End of the Nineteenth Century, Auckland, Penguin Books.

-, 2001. Paradise Reforged: A History of the New Zealanders from the $1880 \mathrm{~s}$ to the Year 2000, Auckland, Allen Lane and The Penguin Press.

BERRY Ruth, 2005 (18/11). Let Women Speak says Sharples, New Zealand Herald (http://subs. nzherald.co.nz/organisation/story.cfm?o_id=265\& ObjectID=10355805, consulté le 31 mars 2012).

DANSEY Harry, 1975. A View of Death, in Michael King (ed.), Te Ao Hurihuri: Aspects of Maoritanga, Auckland, Reed, pp. 173-189.

De l'Estoile, Benoît, 2007. Le goût des Autres : De l'Exposition coloniale aux Arts premiers, Paris, Flammarion.

DurIE Mason, 1999. Marae and Implications for a Modern Māori Psychology, Journal of the Polynesian Society 108 (4), pp. 351-366.

Higgins Rawinia and Paul Meredith, 2011. Tangihanga - death customs - Tangihanga in modern times, Te Ara - the Encyclopedia of New Zealand http://www.TeAra.govt.nz/en/tangihangadeath-customs/5, consulté le 31 mars 2012).

GAGNÉ Natacha, 2008. L'analyse des relations entre minorités et majorités : retour sur la situation néozélandaise à l'aube des années 2000, Anthropologica 50 (1), pp. 101-119.

—, 2011. L'inter-monde de qui ? Analyse d'un « choc de la réalité » chez les Maaori en contexte colonial, in Marie-Blanche Tahon (éd.), Sociologie de l'intermonde: La vie sociale après l'idée de société,
Louvain-la-Neuve, Presses universitaires de Louvain, pp. 153-168.

_, 2013 (sous presse). Being Māori in the City: Indigenous Everyday Life in Auckland, Toronto, University of Toronto Press.

Herewini Te Herekiekie, 2011. Message from the Manager, Te Pānui O Karanga Aotearoa: Karanga Aotearoa Newsletter 4 (1-2) (http://www.tepapa. govt.nz/SiteCollectionDocuments/AboutTePapa/ Repatriation $/ \mathrm{Te} \% 20$ Panui $\% 20 \mathrm{o} \% 20$ Karanga $\% 20$ Aotearoa $\% 20$ Issue $\% 20 \mathrm{No}^{2}$ 204.pdf, consulté le 31 mars 2012).

Hunt Tom, 2011 (9/05). Te Papa team to bring home tattooed heads, The Dominion Post (http://www. stuff.co.nz/dominion-post/news/4977477/Te-Papateam-to-bring-home-tattooed-heads, consulté le 31 mars 2012).

Inimaera Witi, 1972. Pounamu, Pounamu, Auckland, Heinemann.

Jolly Margaret, 2011. Becoming a "New" Museum? Contesting Oceanic Visions at musée du quai Branly, The Contemporary Pacific 23(1), pp. 108139.

JoNEs D. Gareth and Maja I. Whitaker, 2009. Speaking for the dead: the human body in biology and medicine, Farnham, Ashgate.

Karanga Aotearoa, 2012. Karanga Aotearoa Repatriation Programme: Background Document (http://www.mch.govt.nz/files/437839-Te\%20Papa $\% 20$ Repatriation $\% 20$ Programme $\% 20 \mathrm{Jan} \% 202012$. doc, consulté le 31 mars 2012).

Massau Serge, 2011. Paroles d'un autochtone : Entretiens avec le sénateur Richard Ari'ihau Tuheiava, Pape'ete, Haere Pō.

Matenga-Kohu Jayne and Jude Roberts, 2006. Pōwhiri: Rituals of Encounter, Cambridge, New Zealand, Wotz Wot.

McCarthy Conal, 2007. Exhibiting Māori: A History of Colonial Cultures of Display, New York, Berg.

-, 2011. Museums and Māori: Heritage Professionals, Indigenous Collections, Current Practice, Walnut Creek, Left Coast Press.

McIntosh Tracey, 2001. Death, Every Day, in Claudia Bell (ed.), Sociology of Everyday Life in New Zealand, Palmerston North, Dunmore Press, pp. 234251

MeAD Sydney Moko, 1997. Landmarks, bridges and visions: aspects of Maori culture, Wellington, Victoria University Press.

Metge Joan, 1976. The Maoris of New Zealand Rautahi, London, Routledge and Kegan Paul.

—, 1995. New Growth from Old: The Whānau in the Modern World, Wellington, Victoria University Press.

NGata Paratene, 2005. Death, Dying and Grief, in Margot Schwass (ed.), Last Words: Approaches to Death in New Zealand's Cultures and Faiths, 
Wellington, Bridget Williams Books et The Funeral Directors Association of New Zealand, pp. 29-40.

Niezen Ronald, 2010. Public Justice and the Anthropology of Law, Cambridge, Cambridge University Press.

OpPenHeIM Roger, 1973. Maori Death Customs, Wellington, Reed.

Paterson Robert K., 1999. Protecting Taonga: The Cultural Heritage of the New Zealand Maori, International Journal of Cultural Property 8 (1), pp. 108-132.

PICKMERE Arnold, 2002 (5/10). Obituary: Dalvanius Prime, New Zealand Herald (http://www. nzherald.co.nz/nz/news/article.cfm?c_id=1\&objectid =2997633, consulté le 20 mars 2012).

PrÉAUd Martin, 2011. Two Intercultural Stagings with the Yolngu and the Kija: The Representation of Relations, in Barbara Glowczewski et Rosita Henry (eds), The Challenge of Indigenous Peoples: Spectacle or Politics?, Oxford, The Bardwell Press, pp. 83-100.

PRICE Sally, 2011. Au musée des illusions: le rendez-vous manqué du quai Branly, Paris, Éditions Denoël.

Rosenblatt Daniel, 2002. "Titiranti is the Mountain": Representing Maori Community in Auckland, Pacific Studies 25 (1-2), pp. 117-140.

—, 2005. Thinking Outside the Billiard Ball: Cognatic Nationalism and Performing a Maori Public Sphere, Ethnohistory 52 (1), pp. 111-136.

_, 2011. Indigenizing the City and the Future of Maori Culture: The Construction of Community in Auckland as Representation, Experience, and SelfMaking, American Ethnologist 38 (3), pp. 411-429.

SAlmond Anne, 1975. Hui: A Study of Maori Ceremonial Gatherings, Auckland, Reed Books.

Schwartz Dominique, 2012 (27/01). Maori push to preserve tattooed heads, $A B C$ News (http://www. abc.net.au/am/content/2012/s3416604.htm, consulté le 20 mars 2012).

SCHWImmer Éric, 1965. The Cognitive Aspect of Culture Change, Journal of the Polynesian Society 74, pp. 149-181.

—, 1972. Symbolic Competition, Anthropologica XIV (2), pp. 117-155.

Simpson Moira G., 2001. Making Representations: Museums in the Post-Colonial Era, London, Routledge.
SINCLAIR Karen P., 1990. Tangi: Funeral Rituals and the Construction of Maori Identity, in Jocelyn Linnekin and Lin Poyer (eds), Cultural Identity and Ethnicity in the Pacific, Honolulu, University of Hawaii Press, pp. 219-236.

Sissons Jeffrey, 2005. First Peoples: Indigenous Cultures and their Futures, London, Reaktion Books.

—, 2010. Building a House Society: The Reorganization of Maori Communities around Meeting Houses, Journal of the Royal Anthropological Institute 16, pp. 372-386.

TAHANA Yvonne, 2011 (10/05). Maori head service full of French pomp, New Zealand Herald, (http://www. nzherald.co.nz/nz/news/article.cfm?c_id=1\&objectid $=10724531$, consulté le 20 mars 2012).

TAMARAPA Awhina, 2011. Introduction, in Awhina Tamarapa (ed.), Whatu Kākahu / Māori Cloaks, Wellington, Te Papa Press, pp. 9-18.

TAPSELl Paul, 2002. Marae and Tribal Identity in Urban Aotearoa/New Zealand, Pacific Studies 25 (1-2), pp. 141-171.

Tauroa Hiwi et Pat Tauroa, 1986. Te Marae: A Guide to Customs \& Protocol, Auckland, Reed Books.

TE PAPA, s.d.. The Karanga Aotearoa Repatriation Programme, Ressources 1 à 11, Wellington, Te Papa (http://www.tepapa.govt.nz/SiteCollection Documents/AboutTePapa/Repatriation/Karanga $\%$ 20Aotearoa\%20Resources.pdf, consulté le 31 mars 2012).

Museum of New Zealand Te Papa Tongarewa, s.d., Pounamu - Strengthening relationships, Kura Pounamou: Treasured Stone of Aotearoa New Zealand (http://collections.tepapa.govt.nz/exhibitions/ pounamu/segment.aspx?irn=1979, consulté le 31 mars 2012).

Terrell John Edward, Désirée C. J. Wisse et Christopher J. PHILIPP, 2008. Ruatepupuke II - the past and possible future, in Dean Sully (ed.), Decolonising Conservation: Caring for Maori Meeting Houses outside New Zealand, Walnut Creek, Left Coast Press, pp. 89-109.

Walker Ranginui, 2004 (2 éd.). Ka Whawhai Tonu Matou: Struggle Without End, Auckland, Penguin Books. 